\title{
Rich Bifurcation Structure in a Two-Patch Vaccination Model ${ }^{*}$
}

\author{
Diána H. Knipl ${ }^{\dagger}$, Paweł Pilarczyk $k^{\ddagger}$, and Gergely Röst ${ }^{\S}$
}

\begin{abstract}
We show that incorporating spatial dispersal of individuals into a simple vaccination epidemic model may give rise to a model that exhibits rich dynamical behavior. Using an SIVS (susceptibleinfected-vaccinated-susceptible) model as a basis, we describe the spread of an infectious disease in a population split into two regions. In each subpopulation, both forward and backward bifurcations can occur. This implies that for disconnected regions the two-patch system may admit several steady states. We consider traveling between the regions and investigate the impact of spatial dispersal of individuals on the model dynamics. We establish conditions for the existence of multiple nontrivial steady states in the system, and we study the structure of the equilibria. The mathematical analysis reveals an unusually rich dynamical behavior, not normally found in the simple epidemic models. In addition to the disease-free equilibrium, eight endemic equilibria emerge from backward transcritical and saddle-node bifurcation points, forming an interesting bifurcation diagram. Stability of steady states, their bifurcations, and the global dynamics are investigated with analytical tools, numerical simulations, and rigorous set-oriented numerical computations.
\end{abstract}

Key words. backward bifurcation, patch model, equilibrium, numerical simulations, rigorous numerics, computational dynamics, set-oriented computations, ODE, time-1 map, Conley index, Morse decomposition

AMS subject classifications. Primary, 92D30; Secondary, 92-08

DOI. $10.1137 / 140993934$

1. Introduction. The basic reproduction number $\mathcal{R}$ is a central quantity in epidemiology, as it determines the average number of secondary infections caused by a typical infected individual introduced into a wholly susceptible population. In disease transmission models, the reproduction number serves as a threshold for the stability of the disease-free equilibrium

\footnotetext{
* Received by the editors November 3, 2014; accepted for publication (in revised form) by E. Sander March 24, 2015; published electronically June 11, 2015.

http://www.siam.org/journals/siads/14-2/99393.html

${ }^{\dagger}$ Agent-Based Modelling Laboratory, York University, 331 Lumbers, 4700 Keele St., Toronto, ON M3J 1P3, Canada (knipl@yorku.ca), and MTA-SZTE Analysis and Stochastic Research Group, University of Szeged, Aradi vértanúk tere 1, Szeged, Hungary, H-6720 (knipl@math.u-szeged.hu). This author's work was partially supported by the European Union and the State of Hungary, co-financed by the European Social Fund in the framework of TÁMOP 4.2.4. A/2-11-1-2012-0001 "National Excellence Program."

¥Institute of Science and Technology Austria, Am Campus 1, 3400 Klosterneuburg, Austria (pawel.pilarczyk@ ist.ac.at). This author's work was partially supported by the People Programme (Marie Curie Actions) of the European Union's Seventh Framework Programme (FP7/2007-2013) under REA grant agreement 622033, by Fundo Europeu de Desenvolvimento Regional (FEDER) through COMPETE-Programa Operacional Factores de Competitividade (POFC), by the Portuguese national funds through Fundação para a Ciência e a Tecnologia (FCT) in the framework of the research project FCOMP-01-0124-FEDER-010645 (ref. FCT PTDC/MAT/098871/2008), and by the European Research Council through StG 259559 in the framework of the EPIDELAY project.

${ }^{\S}$ Bolyai Institute, University of Szeged, Aradi vértanúk tere 1, Szeged, Hungary, H-6720 (rost@math.u-szeged.hu). This author's work was supported by the European Union and the European Social Fund through project FuturICT. hu (grant TÁMOP-4.2.2.C-11/1/KONV-2012-0013), European Research Council StG 259559, and Hungarian Scientific Research Fund OTKA K109782.
} 
(DFE). The usual situation is that for $\mathcal{R}<1$ the stable DFE is the only equilibrium, but it loses its stability as $\mathcal{R}$ increases through 1 , where a stable positive equilibrium emerges. Such a transition of stability between the DFE and the endemic equilibrium is called forward bifurcation. However, it is possible to have a very different situation at $\mathcal{R}=1$. In case the model undergoes a backward bifurcation at $\mathcal{R}=1$, there is an interval for $\mathcal{R}$ to the left of 1 where multiple positive equilibria (typically one unstable and one stable) coexist with the DFE. The direction of bifurcation is of particular interest from the perspective of controlling the epidemic. If the system exhibits a forward bifurcation at $\mathcal{R}=1$, then for disease eradication it is always sufficient to decrease $\mathcal{R}$ to 1 . On the other hand, in case of backward bifurcation, the presence of a stable endemic equilibrium for $\mathcal{R}<1$ makes it necessary to bring the reproduction number well below 1 to successfully eliminate the infection.

Backward bifurcation has been observed in several studies which consider epidemic models for multiple groups with asymmetry between groups and multiple interaction mechanisms (see $[12,15,16]$ and [14] for an overview). Some simple disease transmission models with vaccination of susceptible individuals are discussed by Kribs-Zaleta and Velasco-Hernández $[19,20]$ and by Brauer [4, 5]. A basic model can be described by the following system of ordinary differential equations:

$$
\begin{aligned}
S^{\prime}(t) & =\Lambda(N(t))-\beta(N(t)) S(t) I(t)-(\mu+\phi) S(t)+\gamma I(t)+\theta V(t), \\
I^{\prime}(t) & =\beta(N(t)) S(t) I(t)+\sigma \beta(N(t)) V(t) I(t)-(\mu+\gamma) I(t), \\
V^{\prime}(t) & =\phi S(t)-\sigma \beta(N(t)) V(t) I(t)-(\mu+\theta) V(t),
\end{aligned}
$$

where $S(t), I(t), V(t)$, and $N(t)$ denote the number of susceptible, infected, or vaccinated individuals and the total population size, respectively, at time $t$. It is assumed that all individuals are born susceptible, which is represented by the birth term $\Lambda$, that is a function of the total population. The parameter $\mu$ is the natural death rate in each class. Disease transmission is modeled by the term $\beta(N) S I$, where the transmission rate $\beta$ is a function of the total population, and the parameters $\phi$ and $\gamma$ stand for the vaccination rate of susceptible individuals and the recovery rate of infected individuals, respectively. It is assumed that vaccination loses effect at rate $\theta$, and $0 \leq \sigma \leq 1$ is introduced to model the phenomenon that vaccination may reduce but not completely eliminate susceptibility to infection. With certain conditions on the birth function $\Lambda$, the system (1.1) can be reduced to two equations by means of the theory of asymptotically autonomous systems (see [23, 36, 37]). For this two-dimensional system, a complete qualitative analysis was carried out in [4], including a condition for the existence of backward bifurcation.

In this paper, we consider a two-patch vaccination model in order to investigate the impact of individuals' mobility on the disease dynamics. Our model generalizes the above presented epidemic model (1.1), as we study the spread of the disease when the population is distributed over two geographically discrete locations, which are connected by instantaneous travel. The aim of this work is to describe steady states, their stability, and their bifurcations in the twopatch model and to reveal how individuals' mobility influences the dynamical behavior. Our results demonstrate that incorporating spatial dispersal of individuals into simple vaccination models can result in rich dynamics; in particular, we show that the stable DFE might coexist with three stable and five unstable nontrivial steady states in the model. By exploring the 
bifurcation structure with analytic and numerical methods, we identify two distinct bifurcation routes from nine to three equilibria.

In disease transmission models, investigating the long time behavior of solutions provides key knowledge for determining final epidemic outcome and identifying adequate intervention measures. However, describing the local and global stability of equilibria, their attracting regions, and bifurcations is rather complex when the corresponding system is highly nonlinear and there are a large number of steady states. A rich bifurcation structure implies that the disease dynamics is sensitive to the model parameters and initial conditions. It also allows a variety of different final epidemic outcomes, which makes disease control and mitigation planning rather challenging. Our findings about the coexistence of multiple stable steady states, having very different levels of infection in the patches, resemble the observed phenomenon of the high variability of hepatitis-B prevalence in different cities, which has been attributed to strongly nonlinear disease dynamics [24].

In addition to the "classical" analysis of equilibria, we conduct a series of set-oriented computations for qualitative analysis of the dynamics using combinatorial and topological methods, as introduced in [1]. Roughly speaking, these computations are carried out for prescribed ranges of parameters and allow us to classify the global dynamics encountered within a given bounded region of the phase space by means of enclosing all the chain recurrent dynamics in isolating neighborhoods and providing information about which connecting orbits between them are possible. The Conley index is used to provide additional information about the recurrent sets found. In this method, all the computations are carried out using interval arithmetic (see [26] for a comprehensive introduction), and the results are mathematically rigorous. This is different than in approximate numerical simulations, which are typically much cheaper but may not provide mathematically reliable results. Since our method is based upon using interval arithmetic and outer approximations, the results are valid for entire ranges of parameters; in particular, proof of continuation of isolating neighborhoods is obtained for the ranges of parameters for which the dynamics has been classified as equivalent. Moreover, this method detects not only attractors, but also unstable invariant sets, usually difficult to find using classical numerical methods. Another advantage of this method is that it provides an automatic proof of the fact that outside of the constructed isolating neighborhoods there is no other recurrent dynamics. In particular, this method provides the certainty that no bounded trajectories in the given phase space have been missed, that there are no periodic solutions except those bounded in the regions found explicitly, and that there are no other fixed points and no other kinds of chain recurrent sets.

The paper is organized as follows. In section 2, we introduce a general disease transmission model with vaccination for epidemic spread in two regions. We discuss a restriction of the model to symmetric regions in section 3, and we investigate the equilibria of the model with analytic methods. In section 4 , we undertake numerical simulations to illustrate the rich bifurcation structure that is encountered in the system. Finally, in section 5 we describe rigorous set-oriented numerical computations conducted for the symmetric model at the parameter ranges for which we found the bifurcations. 
2. The general two-patch model. A general disease transmission model with vaccination for epidemic spread in two regions can be described by the system

$$
\begin{aligned}
S_{1}^{\prime}(t)= & \Lambda_{1}-\beta_{1}\left(N_{1}(t)\right) S_{1}(t) I_{1}(t)-\left(\mu_{1}+\phi_{1}\right) S_{1}(t)+\gamma_{1} I_{1}(t)+\theta_{1} V_{1}(t) \\
& -\alpha_{1}^{S} S_{1}(t)+\alpha_{2}^{S} S_{2}(t), \\
I_{1}^{\prime}(t)= & \beta_{1}\left(N_{1}(t)\right) S_{1}(t) I_{1}(t)+\sigma_{1} \beta_{1}\left(N_{1}(t)\right) V_{1}(t) I_{1}(t)-\left(\mu_{1}+\gamma_{1}\right) I_{1}(t) \\
& -\alpha_{1}^{I} I_{1}(t)+\alpha_{2}^{I} I_{2}(t), \\
V_{1}^{\prime}(t)= & \phi_{1} S_{1}(t)-\sigma_{1} \beta_{1}\left(N_{1}(t)\right) V_{1}(t) I_{1}(t)-\left(\mu_{1}+\theta_{1}\right) V_{1}(t) \\
& -\alpha_{1}^{V} V_{1}(t)+\alpha_{2}^{V} V_{2}(t), \\
S_{2}^{\prime}(t)= & \Lambda_{2}-\beta_{2}\left(N_{2}(t)\right) S_{2}(t) I_{2}(t)-\left(\mu_{2}+\phi_{2}\right) S_{2}(t)+\gamma_{2} I_{2}(t)+\theta_{2} V_{2}(t) \\
& -\alpha_{2}^{S} S_{2}(t)+\alpha_{1}^{S} S_{1}(t), \\
I_{2}^{\prime}(t)= & \beta_{2}\left(N_{2}(t)\right) S_{2}(t) I_{2}(t)+\sigma_{2} \beta_{2}\left(N_{2}(t)\right) V_{2}(t) I_{2}(t)-\left(\mu_{2}+\gamma_{2}\right) I_{2}(t) \\
& -\alpha_{2}^{I} I_{2}(t)+\alpha_{1}^{I} I_{1}(t), \\
V_{2}^{\prime}(t)= & \phi_{2} S_{2}(t)-\sigma_{2} \beta_{2}\left(N_{2}(t)\right) V_{2}(t) I_{2}(t)-\left(\mu_{2}+\theta_{2}\right) V_{2}(t) \\
& -\alpha_{2}^{V} V_{2}(t)+\alpha_{1}^{V} V_{1}(t) .
\end{aligned}
$$

We denote the compartments of susceptible, infected, and vaccinated individuals in region $j(j \in\{1,2\})$ by $S_{j}, I_{j}$, and $V_{j}$, respectively, and the total population $N_{j}$ in region $j$ is obtained as $N_{j}(t)=S_{j}(t)+I_{j}(t)+V_{j}(t)$. The description of the model parameters has been given in section 1 . The subscripts 1 and 2 indicate that the two regions may have different characteristics. We assume that susceptible, infected, and vaccinated individuals in region $j$ travel with rates $\alpha_{j}^{S}, \alpha_{j}^{I}$, and $\alpha_{j}^{V}$ from region $j$ to region $k$, where $j, k \in\{1,2\}$ and $j \neq k$. We obtain some simple results for the system (2.1). The proof of the first proposition is trivial and thus omitted.

Proposition 2.1. Nonnegative initial data give rise to nonnegative solutions in system (2.1).

Proposition 2.2. In system (2.1) there exists a unique DFE when the disease is not present in the populations.

Proof. In the disease-free subspace, system (2.1) reduces to

$$
\begin{aligned}
& S_{1}^{\prime}(t)=\Lambda_{1}-\left(\mu_{1}+\phi_{1}\right) S_{1}(t)+\theta_{1} V_{1}(t)-\alpha_{1}^{S} S_{1}(t)+\alpha_{2}^{S} S_{2}(t), \\
& V_{1}^{\prime}(t)=\phi_{1} S_{1}(t)-\left(\mu_{1}+\theta_{1}\right) V_{1}(t)-\alpha_{1}^{V} V_{1}(t)+\alpha_{2}^{V} V_{2}(t), \\
& S_{2}^{\prime}(t)=\Lambda_{2}-\left(\mu_{2}+\phi_{2}\right) S_{2}(t)+\theta_{2} V_{2}(t)-\alpha_{2}^{S} S_{2}(t)+\alpha_{1}^{S} S_{1}(t), \\
& V_{2}^{\prime}(t)=\phi_{2} S_{2}(t)-\left(\mu_{2}+\theta_{2}\right) V_{2}(t)-\alpha_{2}^{V} V_{2}(t)+\alpha_{1}^{V} V_{1}(t) .
\end{aligned}
$$

We derive the unique positive equilibrium of this system as

$$
\left(\begin{array}{c}
\bar{S}_{1} \\
\bar{V}_{1} \\
\bar{S}_{2} \\
\bar{V}_{2}
\end{array}\right)=\left(\begin{array}{cccc}
\mu_{1}+\phi_{1}+\alpha_{1}^{S} & -\theta_{1} & -\alpha_{2}^{S} & 0 \\
-\phi_{1} & \mu_{1}+\theta_{1}+\alpha_{1}^{V} & 0 & -\alpha_{2}^{V} \\
-\alpha_{1}^{S} & 0 & \mu_{2}+\phi_{2}+\alpha_{2}^{S} & -\theta_{2} \\
0 & -\alpha_{1}^{V} & -\phi_{2} & \mu_{2}+\theta_{2}+\alpha_{2}^{V}
\end{array}\right)^{-1}\left(\begin{array}{c}
\Lambda_{1} \\
0 \\
\Lambda_{2} \\
0
\end{array}\right)
$$


It is straightforward that $\left(\bar{S}_{1}, 0, \bar{V}_{1}, \bar{S}_{2}, 0, \bar{V}_{2}\right)$ is a DFE of system (2.1). Uniqueness follows from the uniqueness of $\left(\bar{S}_{1}, \bar{V}_{1}, \bar{S}_{2}, \bar{V}_{2}\right)$ in $(2.2)$.

Proposition 2.3. Assume that $\alpha_{1}^{S}=\alpha_{1}^{I}=\alpha_{1}^{V}$ and $\alpha_{2}^{S}=\alpha_{2}^{I}=\alpha_{2}^{V}$. Then the total population sizes converge to an equilibrium.

Proof. For the total populations in regions 1 and 2, we derive the following differential equation system:

$$
\begin{aligned}
& N_{1}^{\prime}(t)=\Lambda_{1}-\left(\mu_{1}+\alpha_{1}\right) N_{1}(t)+\alpha_{2} N_{2}(t), \\
& N_{2}^{\prime}(t)=\Lambda_{2}-\left(\mu_{2}+\alpha_{2}\right) N_{2}(t)+\alpha_{1} N_{1}(t),
\end{aligned}
$$

where $\alpha_{1}$ and $\alpha_{2}$ denote the mobility rates of the populations in region 1 and 2, respectively. One can find that the positive equilibrium $\left(K_{1}, K_{2}\right)$ for the populations is given by

$$
\left(\begin{array}{l}
K_{1} \\
K_{2}
\end{array}\right)=\left(\begin{array}{cc}
\mu_{1}+\alpha_{1} & -\alpha_{2} \\
-\alpha_{1} & \mu_{2}+\alpha_{2}
\end{array}\right)^{-1}\left(\begin{array}{l}
\Lambda_{1} \\
\Lambda_{2}
\end{array}\right)
$$

Set $M_{1}(t):=N_{1}(t)-K_{1}, M_{2}(t):=N_{2}(t)-K_{2}$. Then we obtain the linear system

$$
\begin{aligned}
& M_{1}^{\prime}(t)=-\left(\mu_{1}+\alpha_{1}\right) M_{1}(t)+\alpha_{2} M_{2}(t), \\
& M_{2}^{\prime}(t)=-\left(\mu_{2}+\alpha_{2}\right) M_{2}(t)+\alpha_{1} M_{1}(t) .
\end{aligned}
$$

Since $\alpha_{1}$ and $\alpha_{2}$ are nonnegative and $\mu_{1}, \mu_{2}>0$, the zero solution of the last system is asymptotically stable. This implies the asymptotic stability of the positive equilibrium $\left(K_{1}, K_{2}\right)$.

In what follows, we assume that all disease classes within a patch have the same mobility rate, denoted by $\alpha_{1}$ and $\alpha_{2}$ in regions 1 and 2, respectively. Then, using the fact that $S_{1}(t)=N_{1}(t)-I_{1}(t)-V_{1}(t)$ and $S_{2}(t)=N_{2}(t)-I_{2}(t)-V_{2}(t)$, we rewrite $(2.1)_{2},(2.1)_{3},(2.1)_{5}$, and $(2.1)_{6}$ in terms of $N_{j}(t), I_{j}(t)$, and $V_{j}(t)(j \in\{1,2\})$. This four-dimensional system can be considered as a system of nonautonomous differential equations with nonautonomous terms $N_{1}(t)$ and $N_{2}(t)$, which are governed by system (2.3). Then, by Proposition 2.3, we deduce that system (2.1) is asymptotically autonomous with the limiting system

$$
\begin{aligned}
I_{1}^{\prime}(t)= & \beta_{1}\left(K_{1}\right)\left(K_{1}-I_{1}(t)-\left(1-\sigma_{1}\right) V_{1}(t)\right) I_{1}(t)-\left(\mu_{1}+\gamma_{1}\right) I_{1}(t) \\
& -\alpha_{1} I_{1}(t)+\alpha_{2} I_{2}(t), \\
V_{1}^{\prime}(t)= & \phi_{1}\left(K_{1}-I_{1}(t)\right)-\sigma_{1} \beta_{1}\left(K_{1}\right) V_{1}(t) I_{1}(t)-\left(\mu_{1}+\theta_{1}+\phi_{1}\right) V_{1}(t) \\
& -\alpha_{1} V_{1}(t)+\alpha_{2} V_{2}(t), \\
I_{2}^{\prime}(t)= & \beta_{2}\left(K_{2}\right)\left(K_{2}-I_{2}(t)-\left(1-\sigma_{2}\right) V_{2}(t)\right) I_{2}(t)-\left(\mu_{2}+\gamma_{2}\right) I_{2}(t) \\
& -\alpha_{2} I_{2}(t)+\alpha_{1} I_{1}(t), \\
V_{2}^{\prime}(t)= & \phi_{2}\left(K_{2}-I_{2}(t)\right)-\sigma_{2} \beta_{2}\left(K_{2}\right) V_{2}(t) I_{2}(t)-\left(\mu_{2}+\theta_{2}+\phi_{2}\right) V_{2}(t) \\
& -\alpha_{2} V_{2}(t)+\alpha_{1} V_{1}(t) .
\end{aligned}
$$

As the transmission rates $\beta_{1}\left(K_{1}\right)$ and $\beta_{2}\left(K_{2}\right)$ are constants in the system (2.4), going forward we simply write $\beta_{1}$ and $\beta_{2}$. In what follows, we focus on the mathematical analysis of the system (2.4) in the feasible phase space (i.e., in the set of biologically relevant states)

$$
X=\left\{\left(I_{1}, V_{1}, I_{2}, V_{2}\right) \in \mathbb{R}_{+}^{4} \mid I_{1}+V_{1} \leq K_{1}, I_{2}+V_{2} \leq K_{2}\right\} .
$$


As the positive equilibrium $\left(K_{1}, K_{2}\right)$ is globally attracting for the populations in the two regions, we can assume that the two populations have attained their steady states and thus are both constant.

The DFE of the system (2.4) can be obtained as $\left(0, \bar{V}_{1}, 0, \bar{V}_{2}\right)$, where

$$
\left(\begin{array}{l}
\bar{V}_{1} \\
\bar{V}_{2}
\end{array}\right)=\left(\begin{array}{cc}
\mu_{1}+\theta_{1}+\phi_{1}+\alpha_{1} & -\alpha_{2} \\
-\alpha_{1} & \mu_{2}+\theta_{2}+\phi_{2}+\alpha_{2}
\end{array}\right)^{-1}\left(\begin{array}{l}
\phi_{1} K_{1} \\
\phi_{2} K_{2}
\end{array}\right) .
$$

The concept of the next generation matrix (NGM) of an epidemic model was introduced by Diekmann, Heesterbeek, and Metz [10] (elaborated in [9, Chapter 5]) as a matrix whose elements give the number of newly infected individuals in specific categories. To obtain this matrix, we consider the equations of the system that describe the production of new infections and changes in state among infected individuals. In the initial stage of an epidemic, we can assume that system (2.4) is near the $\operatorname{DFE}\left(0, \bar{V}_{1}, 0, \bar{V}_{2}\right)$ and approximate the equations of classes $I_{1}, I_{2}$ with the linear system

$$
\begin{aligned}
& y_{1}^{\prime}(t)=\left(\beta_{1}\left(K_{1}-\left(1-\sigma_{1}\right) \bar{V}_{1}\right)-\left(\mu_{1}+\gamma_{1}+\alpha_{1}\right)\right) y_{1}(t)+\alpha_{2} y_{2}(t), \\
& y_{2}^{\prime}(t)=\left(\beta_{2}\left(K_{2}-\left(1-\sigma_{2}\right) \bar{V}_{2}\right)-\left(\mu_{2}+\gamma_{2}+\alpha_{2}\right)\right) y_{2}(t)+\alpha_{1} y_{1}(t),
\end{aligned}
$$

where $y_{1}, y_{2}: \mathbb{R} \rightarrow \mathbb{R}$. By defining matrices $\mathcal{F}, \mathcal{V} \in \mathbb{R}^{2 \times 2}$ as

$$
\begin{aligned}
& \mathcal{F}=\left(\begin{array}{cc}
\beta_{1}\left(K_{1}-\left(1-\sigma_{1}\right) \bar{V}_{1}\right) & 0 \\
0 & \beta_{2}\left(K_{2}-\left(1-\sigma_{2}\right) \bar{V}_{2}\right)
\end{array}\right), \\
& \mathcal{V}=\left(\begin{array}{cc}
\mu_{1}+\gamma_{1}+\alpha_{1} & -\alpha_{2} \\
-\alpha_{1} & \mu_{2}+\gamma_{2}+\alpha_{2}
\end{array}\right),
\end{aligned}
$$

we obtain the compact form of system (2.6) as

$$
y^{\prime}(t)=(\mathcal{F}-\mathcal{V}) y(t)
$$

The matrix $\mathcal{F}$ can be referred to as the transmission matrix, describing the production of new infections, and $-\mathcal{V}$ is the transition matrix, describing changes in state (see $[9,38]$ for some details). Clearly, $\mathcal{F}$ is a positive matrix - that is, all of its entries are nonnegative - and it is easy to check that $-\mathcal{V}$ is positive-off-diagonal - that is, all entries are nonnegative, possibly except for those at the diagonal. For a square matrix $M$, we define the spectral bound $s(M)$ and the spectral radius $\rho(M)$ by $s(M):=\sup \{\operatorname{Re}(\lambda): \lambda \in \sigma(M)\}, \rho(M):=\sup \{|\lambda|: \lambda \in$ $\sigma(M)\}$, where $\sigma(M)$ denotes the set of eigenvalues of $M$. One can show that $s(-\mathcal{V})<0$, which is equivalent to the statement that $\mathcal{V}$ is invertible and $\mathcal{V}^{-1}$ is a positive matrix (for the proof of the equivalence, see, e.g., [9, Lemma 6.12]).

Following the definition of the NGM from [10], $\mathcal{F} \mathcal{V}^{-1} \in \mathbb{R}^{2 \times 2}$ gives the NGM of the system (2.7) as follows:

$$
N G M=\left(\begin{array}{cc}
\frac{\beta_{1}\left(K_{1}-\left(1-\sigma_{1}\right) \bar{V}_{1}\right)\left(\mu_{2}+\gamma_{2}+\alpha_{2}\right)}{\left(\mu_{1}+\gamma_{1}+\alpha_{1}\right)\left(\mu_{2}+\gamma_{2}+\alpha_{2}\right)-\alpha_{1} \alpha_{2}} & \frac{\alpha_{2} \beta_{1}\left(K_{1}-\left(1-\sigma_{1}\right) \bar{V}_{1}\right)}{\left(\mu_{1}+\gamma_{1}+\alpha_{1}\right)\left(\mu_{2}+\gamma_{2}+\alpha_{2}\right)-\alpha_{1} \alpha_{2}} \\
\frac{\alpha_{1} \beta_{2}\left(K_{2}-\left(1-\sigma_{2}\right) \bar{V}_{2}\right)}{\left(\mu_{1}+\gamma_{1}+\alpha_{1}\right)\left(\mu_{2}+\gamma_{2}+\alpha_{2}\right)-\alpha_{1} \alpha_{2}} & \frac{\beta_{2}\left(K_{2}-\left(1-\sigma_{2}\right) \bar{V}_{2}\right)\left(\mu_{1}+\gamma_{1}+\alpha_{1}\right)}{\left(\mu_{1}+\gamma_{1}+\alpha_{1}\right)\left(\mu_{2}+\gamma_{2}+\alpha_{2}\right)-\alpha_{1} \alpha_{2}}
\end{array}\right) .
$$


The basic reproduction number is defined as the dominant eigenvalue of the NGM (whose existence is guaranteed by the Frobenius-Perron theorem). For our model, we denote this number by $\mathcal{R}_{0}$, and we obtain $\mathcal{R}_{0}=\rho\left(\mathcal{F} \mathcal{V}^{-1}\right)$. We state the following proposition to show that $\mathcal{R}_{0}$ serves as a threshold quantity for the stability of the zero solution of the system (2.7).

Proposition 2.4. The zero solution of $y^{\prime}(t)=(\mathcal{F}-\mathcal{V}) y(t)$ is asymptotically stable if $\mathcal{R}_{0}<1$, and unstable if $\mathcal{R}_{0}>1$.

Proof. We have seen that $\mathcal{F}$ is a positive matrix and $-\mathcal{V}$ is a positive-off-diagonal matrix with $s(-\mathcal{V})<0$. The stability of the zero steady state of $y^{\prime}(t)=(\mathcal{F}-\mathcal{V}) y(t)$ is determined by the sign of $s(\mathcal{F}-\mathcal{V})$, which coincides with the sign of $\rho\left(\mathcal{F} \mathcal{V}^{-1}\right)-1$ (see [11, Theorem A.1]). The equality $\mathcal{R}_{0}=\rho\left(\mathcal{F} \mathcal{V}^{-1}\right)$ completes the proof.

The statement of Proposition 2.4 extends to the nonlinear system (2.4) by the principle of linearized stability.

Theorem 2.5. The DFE of system (2.4) is asymptotically stable if $\mathcal{R}_{0}<1$, and unstable if $\mathcal{R}_{0}>1$.

Proposition 2.6. If $\beta_{1}=0$ and $\beta_{2}=0$, then there exists only one steady state, the DFE, which is globally asymptotically stable.

Proof. If the transmission rates vanish in both regions, then system (2.4) reduces to the linear system

$$
\begin{aligned}
I_{1}^{\prime}(t) & =-\left(\mu_{1}+\gamma_{1}\right) I_{1}(t)-\alpha_{1} I_{1}(t)+\alpha_{2} I_{2}(t), \\
V_{1}^{\prime}(t) & =\phi_{1}\left(K_{1}-I_{1}(t)\right)-\left(\mu_{1}+\theta_{1}+\phi_{1}\right) V_{1}(t)-\alpha_{1} V_{1}(t)+\alpha_{2} V_{2}(t), \\
I_{2}^{\prime}(t) & =-\left(\mu_{2}+\gamma_{2}\right) I_{2}(t)-\alpha_{2} I_{2}(t)+\alpha_{1} I_{1}(t), \\
V_{2}^{\prime}(t) & =\phi_{2}\left(K_{2}-I_{2}(t)\right)-\left(\mu_{2}+\theta_{2}+\phi_{2}\right) V_{2}(t)-\alpha_{2} V_{2}(t)+\alpha_{1} V_{1}(t),
\end{aligned}
$$

which has only one equilibrium $\left(0, \bar{V}_{1}, 0, \bar{V}_{2}\right)$. It is easy to see that $\lim _{t \rightarrow \infty}\left(I_{1}(t)+I_{2}(t)\right)=0$, and, as solutions obviously remain nonnegative for nonnegative initial data, it follows that $\lim _{t \rightarrow \infty} I_{1}(t)=0$ and $\lim _{t \rightarrow \infty} I_{2}(t)=0$. Let us now investigate the solutions of the system

$$
\begin{aligned}
& W_{1}^{\prime}(t)=\phi_{1} K_{1}-\left(\mu_{1}+\theta_{1}+\phi_{1}\right) W_{1}(t)-\alpha_{1} W_{1}(t)+\alpha_{2} W_{2}(t), \\
& W_{2}^{\prime}(t)=\phi_{2} K_{2}-\left(\mu_{2}+\theta_{2}+\phi_{2}\right) W_{2}(t)-\alpha_{2} W_{2}(t)+\alpha_{1} W_{1}(t) .
\end{aligned}
$$

We introduce the change of variables $Z_{1}(t):=W_{1}(t)-\bar{V}_{1}$ and $Z_{2}(t):=W_{2}(t)-\bar{V}_{2}$ to shift the equilibrium $\left(\bar{V}_{1}, \bar{V}_{2}\right)$ to the origin. Then the asymptotic stability of the zero solution of the system

$$
\begin{aligned}
& Z_{1}^{\prime}(t)=-\left(\mu_{1}+\theta_{1}+\phi_{1}+\alpha_{1}\right) Z_{1}(t)+\alpha_{2} Z_{2}(t), \\
& Z_{2}^{\prime}(t)=-\left(\mu_{2}+\theta_{2}+\phi_{2}+\alpha_{2}\right) Z_{2}(t)+\alpha_{1} Z_{1}(t)
\end{aligned}
$$

implies that solutions of $(2.9)$ converge to $\left(\bar{V}_{1}, \bar{V}_{2}\right)$. Since $\lim _{t \rightarrow \infty} I_{1}(t)=0$ and $\lim _{t \rightarrow \infty} I_{2}(t)=$ 0 , we can consider $(2.8)_{2}$ and $(2.8)_{4}$ as nonautonomous equations with nonautonomous terms $I_{1}(t)$ and $I_{2}(t)$ and conclude that (2.9) serves as the limiting system of $(2.8)_{2}$ and $(2.8)_{4}$. Then, using the theory of asymptotically autonomous systems [23, 36, 37], we conclude that $\lim _{t \rightarrow \infty} V_{1}(t)=\bar{V}_{1}$ and $\lim _{t \rightarrow \infty} V_{2}(t)=\bar{V}_{2}$.

We note that system (2.4) is equivalent to (2.9) in the disease-free subspace, which implies the following result. 
Proposition 2.7. In the disease-free subspace, there exists a unique equilibrium $\left(\bar{V}_{1}, \bar{V}_{2}\right)$, which is globally asymptotically stable.

3. The case of symmetric regions. In what follows we assume that the regions are symmetric in the model parameters. This assumption enables us to focus our attention on the impact of mobility on the dynamics. By dropping parameter indices, we obtain the system

$$
\begin{aligned}
I_{1}^{\prime}(t)= & \beta\left(K-I_{1}(t)-(1-\sigma) V_{1}(t)\right) I_{1}(t)-(\mu+\gamma) I_{1}(t) \\
& -\alpha I_{1}(t)+\alpha I_{2}(t), \\
V_{1}^{\prime}(t)= & \phi\left(K-I_{1}(t)\right)-\sigma \beta V_{1}(t) I_{1}(t)-(\mu+\theta+\phi) V_{1}(t) \\
& -\alpha V_{1}(t)+\alpha V_{2}(t),
\end{aligned}
$$

$$
\begin{aligned}
I_{2}^{\prime}(t)= & \beta\left(K-I_{2}(t)-(1-\sigma) V_{2}(t)\right) I_{2}(t)-(\mu+\gamma) I_{2}(t) \\
& -\alpha I_{2}(t)+\alpha I_{1}(t), \\
V_{2}^{\prime}(t)= & \phi\left(K-I_{2}(t)\right)-\sigma \beta V_{2}(t) I_{2}(t)-(\mu+\theta+\phi) V_{2}(t) \\
& -\alpha V_{2}(t)+\alpha V_{1}(t)
\end{aligned}
$$

for the spread of the disease in two symmetric regions. Note that if $\sigma=1$, which means that the vaccine is completely ineffective, then the model is equivalent to the standard SIS (susceptible-infected-susceptible) model. The effect of spatial dispersal on the dynamics in SIS-based models has been studied extensively in the literature (see, for instance, $[3,18,39,40]$ and many others); hence we don't investigate this case any further in our paper.

As in section 2, we learn that there is a unique DFE in the model $(\mathrm{T})$, where the formula for the steady state in the vaccinated classes is given by

$$
\bar{V}=\frac{\phi K}{\mu+\theta+\phi} .
$$

It follows that the NGM takes the form

$$
N G M=\frac{\beta(K-(1-\sigma) \bar{V})}{(\mu+\gamma+\alpha)^{2}-\alpha^{2}}\left(\begin{array}{cc}
\mu+\gamma+\alpha & \alpha \\
\alpha & \mu+\gamma+\alpha
\end{array}\right),
$$

and an explicit formula arises for the reproduction number, as

$$
\mathcal{R}_{0}=\frac{\beta K}{\mu+\gamma} \cdot \frac{\mu+\theta+\sigma \phi}{\mu+\theta+\phi} .
$$

By Theorem 2.5, $\mathcal{R}_{0}$ serves as a threshold for the stability of the DFE.

Equilibria of the model $(\mathrm{T})$ are solutions of the four-dimensional system

$$
\begin{aligned}
& 0=\beta\left(K-\hat{I}_{1}-(1-\sigma) \hat{V}_{1}\right) \hat{I}_{1}-(\mu+\gamma) \hat{I}_{1}-\alpha \hat{I}_{1}+\alpha \hat{I}_{2}, \\
& 0=\phi\left(K-\hat{I}_{1}\right)-\sigma \beta \hat{V}_{1} \hat{I}_{1}-(\mu+\theta+\phi) \hat{V}_{1}-\alpha \hat{V}_{1}+\alpha \hat{V}_{2}, \\
& 0=\beta\left(K-\hat{I}_{2}-(1-\sigma) \hat{V}_{2}\right) \hat{I}_{2}-(\mu+\gamma) \hat{I}_{2}-\alpha \hat{I}_{2}+\alpha \hat{I}_{1}, \\
& 0=\phi\left(K-\hat{I}_{2}\right)-\sigma \beta \hat{V}_{2} \hat{I}_{2}-(\mu+\theta+\phi) \hat{V}_{2}-\alpha \hat{V}_{2}+\alpha \hat{V}_{1} .
\end{aligned}
$$


From $(3.2)_{1}$ and $(3.2)_{3}$, we obtain the formulas

$$
\begin{aligned}
& \hat{V}_{1}=\frac{K-\hat{I}_{1}}{1-\sigma}+\frac{(\mu+\gamma+\alpha) \hat{I}_{1}-\alpha \hat{I}_{2}}{\beta \hat{I}_{1}(\sigma-1)}, \\
& \hat{V}_{2}=\frac{K-\hat{I}_{2}}{1-\sigma}+\frac{(\mu+\gamma+\alpha) \hat{I}_{2}-\alpha \hat{I}_{1}}{\beta \hat{I}_{2}(\sigma-1)}
\end{aligned}
$$

for $\hat{V}_{1}$ and $\hat{V}_{2}$ whenever $\hat{I}_{1} \neq 0$ and $\hat{I}_{2} \neq 0$, respectively. In what follows, an equilibrium of the model $(\mathrm{T})$ will be denoted by $E=\left(\hat{I}_{1}, \hat{V}_{1}, \hat{I}_{2}, \hat{V}_{2}\right)$, which can also represent the DFE $(0, \bar{V}, 0, \bar{V})$.

3.1. Disconnected regions. In the special case when $\alpha=0$ and the two regions are disconnected, we denote the model $(\mathrm{T})$ by $\left(\mathrm{T}_{0}\right)$. The notation $E^{0}=\left(\left(\hat{I}_{1}\right)^{0},\left(\hat{V}_{1}\right)^{0},\left(\hat{I}_{2}\right)^{0}\right.$, $\left.\left(\hat{V}_{2}\right)^{0}\right)$ will be used for a steady state of the model $\left(\mathrm{T}_{0}\right)$.

In the model $\left(\mathrm{T}_{0}\right)$, the first two equations of (3.2) decouple from the last two, and $\hat{I}_{1}$ and $\hat{I}_{2}$ arise independently from one another as the solutions of the system

$$
\begin{aligned}
& 0=\beta(K-\hat{I}-(1-\sigma) \hat{V}) \hat{I}-(\mu+\gamma) \hat{I}, \\
& 0=\phi(K-\hat{I})-\sigma \beta \hat{V} \hat{I}-(\mu+\theta+\phi) \hat{V} .
\end{aligned}
$$

Investigating this system, given by (1.1), was part of the analysis of the one-patch model in [4]. More precisely, in terms of the reproduction number $R(\phi)$ of (1.1) and the condition for the existence of backward bifurcation

$$
\sigma(1-\sigma)(\mu+\gamma) \phi>(\mu+\theta+\sigma \phi)^{2},
$$

full characterization was given for the solutions. We note that the one-patch model in [4] and our model ( $\mathrm{T}$ ) have the same reproduction numbers; i.e., $R(\phi)=\mathcal{R}_{0}$. Thus, here we summarize the results of [4] in terms of $\mathcal{R}_{0}$, but first we give the following remark.

Remark 1. If $\sigma=0$ (the vaccine provides perfect protection), then the condition (c) for backward bifurcation cannot be satisfied. Hence, we have $\sigma \neq 0$ whenever the condition (c) holds true. For the case when the condition (c) doesn't hold, we allow $\sigma \geq 0$.

There is a $\operatorname{DFE}(\hat{I}, \hat{V})=\left(0, \frac{\phi K}{\mu+\theta+\phi}\right)$. In an endemic equilibrium with $\hat{I}>0$, the $V$ component is given by the formula $\hat{V}=\frac{\beta(K-\hat{I}-(\mu+\gamma))}{\beta(1-\sigma)}$, and $\hat{I}$ arises as the solution of $A \hat{I}^{2}+$ $B \hat{I}+C=0$, where

$$
\begin{aligned}
& A=\sigma \beta, \\
& B=(\mu+\theta+\sigma \phi)+\sigma(\mu+\gamma)-\sigma \beta K, \\
& C=\frac{(\mu+\gamma)(\mu+\theta+\phi)}{\beta}-(\mu+\theta+\sigma \phi) K .
\end{aligned}
$$

We denote the solutions of the steady-state equation by

$$
(\breve{I})^{1}=\frac{-B-\sqrt{B^{2}-4 A C}}{2 A}, \quad(\breve{I})^{2}=\frac{-B+\sqrt{B^{2}-4 A C}}{2 A} .
$$


Define the critical value $0<\mathcal{R}_{c}<1$ for the reproduction number as

$$
\mathcal{R}_{c}=\frac{\mu+\theta+\sigma \phi}{\mu+\theta+\phi} \cdot \frac{\sigma(\mu+\gamma)+2 \sqrt{\sigma(1-\sigma)(\mu+\gamma) \phi}-(\mu+\theta+\sigma \phi)}{\sigma(\mu+\gamma)} .
$$

Then the following statements hold for the steady-state equation:

(i) if $\mathcal{R}_{0}<\mathcal{R}_{c}$, then $\hat{I}=0$ is the only solution;

(ii) in the interval $\left(\mathcal{R}_{c}, 1\right)$ there exist exactly three solutions $0,(\breve{I})^{1}$, and $(\breve{I})^{2}$ if (c) holds, and one solution 0 otherwise;

(iii) if $\mathcal{R}_{0}=\mathcal{R}_{c}$ or $\mathcal{R}_{0}=1$, there exist exactly two solutions 0 and $(\breve{I})^{2}$ if (c) holds, and one solution 0 otherwise;

(iv) there exist exactly two solutions 0 and $(\breve{I})^{2}$ if $\mathcal{R}_{0}>1$.

The steady states of the two-patch model $\left(\mathrm{T}_{0}\right)$ are characterized in the next proposition. We recall that the two regions are disconnected, and hence equilibria of each region are attained independently of the dynamics of the other region.

Proposition 3.1. If the condition (c) is satisfied, then the model $\left(\mathrm{T}_{0}\right)$ has one equilibrium with $\left(\hat{I}_{1}\right)^{0}=\left(\hat{I}_{2}\right)^{0}=0$ if $\mathcal{R}_{0}<\mathcal{R}_{c}$, nine equilibria with $\left(\hat{I}_{1}\right)^{0},\left(\hat{I}_{2}\right)^{0} \in\left\{0,(\breve{I})^{1},(\breve{I})^{2}\right\}$ if $\mathcal{R}_{0} \in$ $\left(\mathcal{R}_{c}, 1\right)$, and four equilibria with $\left(\hat{I}_{1}\right)^{0},\left(\hat{I}_{2}\right)^{0} \in\left\{0,(\breve{I})^{2}\right\}$ if either $\mathcal{R}_{0}=\mathcal{R}_{c}$ or $\mathcal{R}_{0} \geq 1$. If (c) does not hold, then for $\mathcal{R}_{0} \leq 1$ only the DFE exists, and there are four equilibria with $\left(\hat{I}_{1}\right)^{0},\left(\hat{I}_{2}\right)^{0} \in\left\{0,(\breve{I})^{2}\right\}$ when $\mathcal{R}_{0}>1$.

The stability of these equilibria can be investigated by making use of the results for the one-patch model. Following [4], we claim that in the one-patch model an equilibrium with $\hat{I}=0$ is stable if $\mathcal{R}_{0}<1$ and unstable if $\mathcal{R}_{0}>1$; moreover, $\hat{I}=(\breve{I})^{1}$ is unstable and $\hat{I}=(\breve{I})^{2}$ is stable, where they exist. It is thus clear that an equilibrium $E^{0}=\left(\left(\hat{I}_{1}\right)^{0},\left(\hat{V}_{1}\right)^{0},\left(\hat{I}_{2}\right)^{0},\left(\hat{V}_{2}\right)^{0}\right)$ of the model $\left(\mathrm{T}_{0}\right)$ is stable if and only if both $\left(\left(\hat{I}_{1}\right)^{0},\left(\hat{V}_{1}\right)^{0}\right)$ and $\left(\left(\hat{I}_{2}\right)^{0},\left(\hat{V}_{2}\right)^{0}\right)$ are stable in the one-patch model. In light of these conclusions, we can also describe bifurcations of fixed points in $\left(\mathrm{T}_{0}\right)$. The results of the next propositions are also illustrated in Figure 1.

Proposition 3.2. If $\mathcal{R}_{0}<\mathcal{R}_{c}$, then only the DFE exists in the model $\left(\mathrm{T}_{0}\right)$, and it is asymptotically stable. At $\mathcal{R}_{0}=\mathcal{R}_{c}$, four fully endemic steady states with $\left(\hat{I}_{1}\right)^{0},\left(\hat{I}_{2}\right)^{0} \in\left\{(\breve{I})^{1},(\breve{I})^{2}\right\}$ bifurcate from a triple saddle-node bifurcation point; that is, one stable and three unstable positive equilibria arise. In addition, from two saddle-node bifurcation points there also emerge two pairs of partially endemic steady states. If $\mathcal{R}_{c}<\mathcal{R}_{0}<1$, then an equilibrium is unstable if $\left(\hat{I}_{1}\right)^{0}=(\breve{I})^{1}$ or $\left(\hat{I}_{2}\right)^{0}=(\breve{I})^{1}$, and asymptotically stable otherwise. As $\mathcal{R}_{0}$ crosses 1 , six nontrivial steady states disappear. Two stable equilibria $\left(0,(\breve{I})^{2}\right),\left((\breve{I})^{2}, 0\right)$ lose their stability as each undergoes a backward transcritical bifurcation. Triple backward transcritical bifurcation describes the situation when three unstable nontrivial equilibria bifurcate into the stable DFE such that the DFE becomes unstable. For $\mathcal{R}_{0}>1$, the equilibria with infected components $(0,0),\left(0,(\breve{I})^{2}\right)$, and $\left((\breve{I})^{2}, 0\right)$ are unstable, and the equilibrium where $\left(\left(\hat{I}_{1}\right)^{0},\left(\hat{I}_{2}\right)^{0}\right)=\left((\breve{I})^{2},(\breve{I})^{2}\right)$ is asymptotically stable.

Remark 2. By excluding periodic solutions, it was shown in [4] that every solution of the one-patch model converges to an equilibrium. In the two-patch model $\left(\mathrm{T}_{0}\right)$ for disconnected regions, the dynamics of the two regions are independent of one another; thus there are no periodic solutions, and every solution approaches an equilibrium. Since we know the possible equilibria and their domains of attraction if there is more than one equilibrium, it follows that we have a complete understanding of the global behavior of the model $\left(\mathrm{T}_{0}\right)$. 


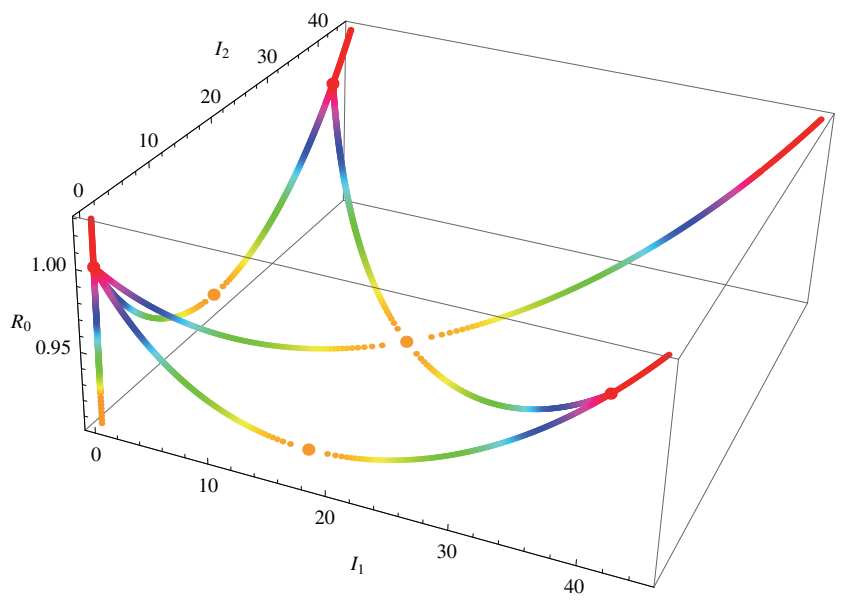

(a) Three-dimensional plot.

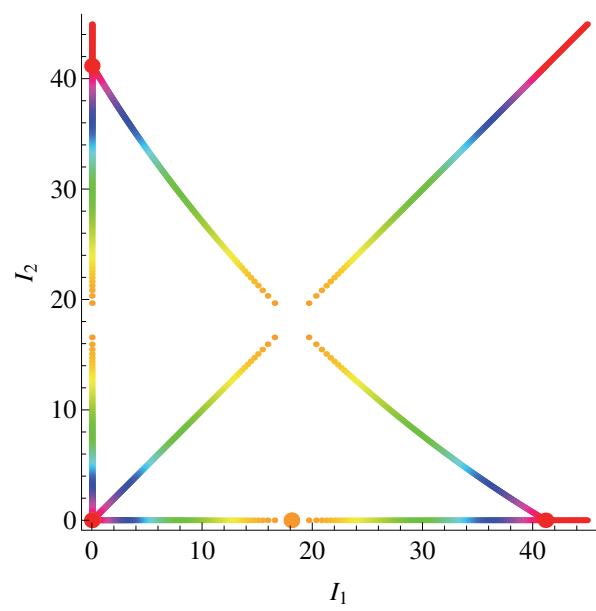

(b) Two-dimensional plot.

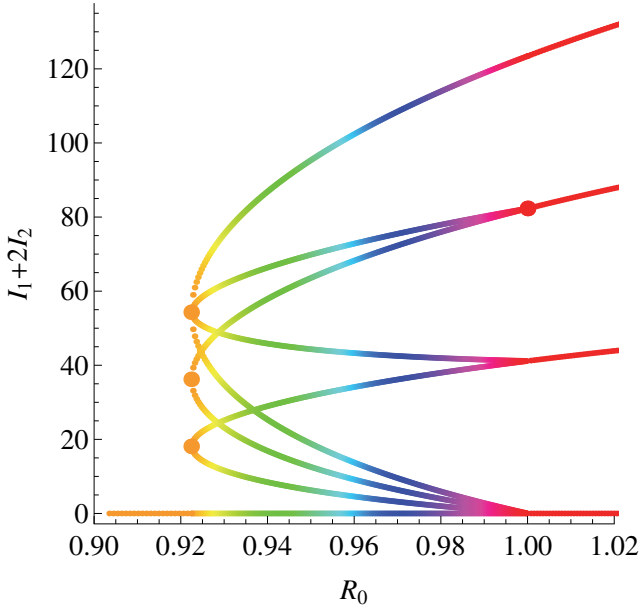

(c) Bifurcation diagram.

Figure 1. Equilibria of the model $\left(\mathrm{T}_{0}\right)$ for $\mathcal{R}_{0} \in(0.9,1.02)$. Color changes from orange through yellow, green, blue, and violet to red as $\mathcal{R}_{0}$ increases from $\mathcal{R}_{c}$ to 1 . Steady states for $\mathcal{R}_{0}<\mathcal{R}_{c}$ and for $\mathcal{R}_{0}>1$ are depicted with orange and red, respectively. At $\mathcal{R}_{0}=\mathcal{R}_{c}$, two large orange dots denote the saddle-node bifurcation points along the $I_{1}$ and $I_{2}$ axes, and the third orange dot indicates the triple saddle-node bifurcation point (one stable and three unstable branches) in the diagonal. At $\mathcal{R}_{0}=1$, the system undergoes a triple backward transcritical bifurcation of the DFE (three unstable steady states bifurcate into a stable equilibrium), and two backward transcritical bifurcations of stable boundary equilibria (large red dots). Parameters were set as $K=100, \mu=0.1, \gamma=12, \theta=0.5, \sigma=0.2, \phi=16$. This makes $\mathcal{R}_{c} \approx 0.9224$, and the condition (c) for multiple nontrivial equilibria is also satisfied.

3.2. Connected regions. We return to the model ( $\mathrm{T})$ and assume that $\alpha$ is positive; i.e., the regions are connected by transportation. The existence of a unique DFE has been proved, so now we focus on finding endemic equilibria $E=\left(\hat{I}_{1}, \hat{V}_{1}, \hat{I}_{2}, \hat{V}_{2}\right)$ with $\hat{I}_{1}>0$ and $\hat{I}_{2}>0$. Note that there is no partially endemic equilibrium; i.e., $\hat{I}_{j}>0, \hat{I}_{k}=0(j, k \in\{1,2\}, j \neq k)$ is not possible since $\hat{I}_{1}=0$ implies $\hat{I}_{2}=0$ and vice versa. By substituting $\hat{V}_{1}$ and $\hat{V}_{2}$ (derived 
in (3.3)) into $(3.2)_{2}$ and $(3.2)_{4}$, it follows from straightforward calculations that

$$
\begin{aligned}
& 0=\frac{1}{\beta \hat{I}_{1} \hat{I}_{2}(\sigma-1)}\left(\alpha^{2}\left(\hat{I}_{1}^{2}-\hat{I}_{2}^{2}\right)+\alpha\left(\hat{I}_{1}-\hat{I}_{2}\right) \hat{I}_{2}\left(\mu+\phi+\beta \hat{I}_{1}(1+\sigma)+\theta\right)\right. \\
& \left.+\hat{I}_{1} \hat{I}_{2} \beta\left(A \hat{I}_{1}^{2}+B \hat{I}_{1}+C\right)\right), \\
& 0=\frac{1}{\beta \hat{I}_{1} \hat{I}_{2}(\sigma-1)}\left(\alpha^{2}\left(\hat{I}_{2}^{2}-\hat{I}_{1}^{2}\right)+\alpha\left(\hat{I}_{2}-\hat{I}_{1}\right) \hat{I}_{1}\left(\mu+\phi+\beta \hat{I}_{2}(1+\sigma)+\theta\right)\right. \\
& \left.+\hat{I}_{1} \hat{I}_{2} \beta\left(A \hat{I}_{2}^{2}+B \hat{I}_{2}+C\right)\right),
\end{aligned}
$$

where $A, B$, and $C$ were defined in (3.4). Similar to the case of disconnected regions, here we again characterize the number of endemic equilibria in terms of $\mathcal{R}_{0}$ and the condition (c). We note that the coefficient $C$ can be expressed by the relation $\beta C=\left(1-\mathcal{R}_{0}\right)(\mu+\gamma)(\mu+\theta+\phi)$ and remark that (c) is equivalent to the condition that $B<0$ holds when $\beta$ is set to satisfy $C=0$. First, we focus our attention on the case when (c) does not hold.

Proposition 3.3. Assume that the condition (c) does not hold. Then there exist no endemic equilibria in the model $(\mathrm{T})$ if $\mathcal{R}_{0} \leq 1$, and there is a unique endemic equilibrium if $\mathcal{R}_{0}>1$. For the endemic equilibrium, $\hat{I}_{1}=\hat{I}_{2}=\frac{-B+\sqrt{B^{2}-4 A C}}{2 A}$.

Proof. We note that $A$ is positive. If $\mathcal{R}_{0} \leq 1$, or equivalently $C \geq 0$, then $B \geq 0$ also holds, since $\sigma(1-\sigma)(\mu+\gamma) \phi \leq(\mu+\theta+\sigma \phi)^{2}$ implies $B \geq 0$ at $\mathcal{R}_{0}=1$ and $B$ increases as $\beta$ decreases. Defining

$$
f(x)=A x^{2}+B x+C,
$$

it is thus easy to see that $f(x)>0$ whenever $x>0$ and $\mathcal{R}_{0} \leq 1$.

We show by the method of contradiction that no endemic equilibria exist if $\mathcal{R}_{0} \leq 1$. Let us assume that $\hat{I}_{1}=\hat{I}_{2}$ holds for a positive solution of the system (3.5). Then this system reduces to two equations as $f\left(\hat{I}_{1}\right)=0$ and $f\left(\hat{I}_{2}\right)=0$, which means that $f$ has at least one zero on $(0, \infty)$, a contradiction. On the other hand, if $\hat{I}_{1} \neq \hat{I}_{2}$ in the endemic equilibrium, then we can assume without loss of generality that $\hat{I}_{1}>\hat{I}_{2}$ holds. From $(3.5)_{1}$, the relation $f\left(\hat{I}_{1}\right)<0$ follows, but this is again impossible since $f(x)>0$ holds if $x$ is positive.

We claim that no positive equilibrium with $\hat{I}_{1} \neq \hat{I}_{2}$ exists if $\mathcal{R}_{0}>1$. Indeed, if $\hat{I}_{1}>\hat{I}_{2}>0$, then to satisfy (3.5) the inequalities $f\left(\hat{I}_{1}\right)<0$ and $f\left(\hat{I}_{2}\right)>0$ should hold. Because $A$ is positive and $C$ is negative, the equation $f(x)=0$ has a single zero on $(0, \infty)$, and thus there is no $\hat{I}_{1}$ and $\hat{I}_{2}$ such that $\hat{I}_{1}>\hat{I}_{2}>0, f\left(\hat{I}_{1}\right)<0$, and $f\left(\hat{I}_{2}\right)>0$ are satisfied. If we look for endemic equilibria where $\hat{I}_{1}=\hat{I}_{2}$, then from (3.5) and the uniqueness of the positive solution of $f(x)=0$ we get that the formula $\frac{-B+\sqrt{B^{2}-4 A C}}{2 A}$ gives $\hat{I}_{1}$ and $\hat{I}_{2}$ in the unique positive equilibrium.

Notation 1. We remark that by (3.3) it follows that $\hat{V}_{1}=\hat{V}_{2}$ holds whenever $\hat{I}_{1}=\hat{I}_{2}$; i.e., an equilibrium with $\hat{I}_{1}=\hat{I}_{2}$ is a "symmetric equilibrium." On the other hand, we can refer to an equilibrium with $\hat{I}_{1} \neq \hat{I}_{2}$ as a "nonsymmetric equilibrium."

Before proceeding with the stability analysis, we investigate the impact of condition (c) on the bifurcation structure. The following lemma is given for later use.

Lemma 3.4. Assume that condition (c) holds and that coefficients $A, B$, and $C$ are given as in (3.4). Then,

$$
f(x)=A x^{2}+B x+C=0
$$


has one positive solution if $\mathcal{R}_{0} \geq 1$, two distinct positive solutions if $\mathcal{R}_{0} \in\left(\mathcal{R}_{c}, 1\right)$, one positive solution if $\mathcal{R}_{0}=\mathcal{R}_{c}$, and no positive solutions if $\mathcal{R}_{0}<\mathcal{R}_{c}$.

Proof. We recall that the relation $\beta C=\left(1-\mathcal{R}_{0}\right)(\mu+\gamma)(\mu+\theta+\phi)$ holds. The equation $f(x)=0$ has exactly one positive solution if $\mathcal{R}_{0}>1$, as $A>0$ and $C<0$ imply the existence of two nonzero roots of opposite sign. Condition (c) yields $B<0$ when $\beta$ is set to satisfy $C=0$. At $\mathcal{R}_{0}=1$, we have $C=0$ and thus $B<0$, so $x=-B / A$ is the only positive solution. We also get $B^{2}-4 A C=B^{2}>0$ at $\mathcal{R}_{0}=1$; hence due to the continuous dependence of $B$ and $\mathcal{R}_{0}$ on $\beta$, there must be an interval to the left of $\mathcal{R}_{0}=1$ where $B<0, C>0$, and $B^{2}-4 A C>0$ hold, and hence there exist two positive roots. This interval is given as $\left(\mathcal{R}_{c}, 1\right)$, since at $\mathcal{R}_{0}=\mathcal{R}_{c}$ we have $B^{2}-4 A C=0, B<0$, and $C>0$, so the equation $f(x)=0$ has a single positive solution $x=-B / 2 A$ with double multiplicity. It is easy to see that $B^{2}-4 A C$ decreases as we further decrease $\beta$, which implies that no real roots exist for $\mathcal{R}_{0}<\mathcal{R}_{c}$.

Proposition 3.5. Assume that condition (c) holds. If either $\mathcal{R}_{0}=\mathcal{R}_{c}$ or $\mathcal{R}_{0} \geq 1$, then there is a unique endemic equilibrium $\left(\hat{I}_{1}, \hat{V}_{1}, \hat{I}_{2}, \hat{V}_{2}\right)$ in the model $(\mathrm{T})$, for which $\hat{I}_{1}=\hat{I}_{2}=(\breve{I})^{2}$. There are no endemic equilibria for $\mathcal{R}_{0}<\mathcal{R}_{c}$.

Proof. First, we show that if $\mathcal{R}_{0}$ is outside of the interval $\left(\mathcal{R}_{c}, 1\right)$, then there exist no equilibria such that $\hat{I}_{1} \neq \hat{I}_{2}$. We consider steady-state solutions where $\hat{I}_{1}>\hat{I}_{2}>0$; the case when $\hat{I}_{2}>\hat{I}_{1}>0$ can be treated similarly. We derive from (3.5) that $f\left(\hat{I}_{1}\right)<0$ and $f\left(\hat{I}_{2}\right)>0$ should hold for the equilibrium. The previous lemma implies that $f(x)=0$ has at most one positive solution if $\mathcal{R}_{0} \leq \mathcal{R}_{c}$ or $\mathcal{R}_{0} \geq 1$. It thus follows that the inequalities $f\left(\hat{I}_{1}\right)<0$ and $f\left(\hat{I}_{2}\right)>0$ can never be satisfied if $\hat{I}_{1}>\hat{I}_{2}>0$.

Next, we look for symmetric endemic equilibria $(\hat{I}, \hat{V}, \hat{I}, \hat{V})$ in the cases when $\mathcal{R}_{0} \leq \mathcal{R}_{c}$ and $\mathcal{R}_{0} \geq 1$. If $\hat{I}_{1}=\hat{I}_{2}=\hat{I}$, then the system (3.5) reduces to the single equation

$$
f(\hat{I})=A \hat{I}^{2}+B \hat{I}+C=0 .
$$

The statement of the proposition immediately follows from Lemma 3.4. We remark that the positive root, whenever it is unique, is given as $\hat{I}=\frac{-B+\sqrt{B^{2}-4 A C}}{2 A}=(\breve{I})^{2}$.

We learned from Propositions 3.3 and 3.5 that the existence of nonsymmetric equilibria is possible only if condition (c) is satisfied and $\mathcal{R}_{0} \in\left(\mathcal{R}_{c}, 1\right)$. In what follows, we investigate steady states in this region of the parameter space. The next result concerns symmetric endemic equilibria in the case when (c) holds and $\mathcal{R}_{c}<\mathcal{R}_{0}<1$.

Proposition 3.6. Assume that condition (c) holds. If $\mathcal{R}_{c}<\mathcal{R}_{0}<1$, then the model (T) has exactly two positive symmetric equilibria. For these equilibria, $\hat{I}_{1}=\hat{I}_{2}$ and $\hat{I}_{1}, \hat{I}_{2} \in\left\{(\breve{I})^{1},(\breve{I})^{2}\right\}$.

Proof. For symmetric equilibria, the system (3.5) again reduces to $f(\hat{I})=0$. Conditions (c) and $\mathcal{R}_{0} \in\left(\mathcal{R}_{c}, 1\right)$ are equivalent to $A>0, B<0, C>0$, and $B^{2}-4 A C>0$. We refer to Lemma 3.4 to know that the equation $A \hat{I}^{2}+B \hat{I}+C=0$ has two positive distinct solutions $(\breve{I})^{1}$ and $(\breve{I})^{2}$.

Next, we summarize our results about symmetric endemic equilibria, and we characterize their (local) stability.

Proposition 3.7. In the model $(\mathrm{T})$, the symmetric equilibrium where $\hat{I}=(\breve{I})^{2}$ is locally asymptotically stable where it exists: on $\mathcal{R}_{0} \in(1, \infty)$, and also on $\mathcal{R}_{0} \in\left(\mathcal{R}_{c}, 1\right]$ when (c) holds. The symmetric equilibrium where $\hat{I}=(\breve{I})^{1}$ is unstable where it exists: on $\mathcal{R}_{0} \in\left(\mathcal{R}_{c}, 1\right)$ when (c) holds. 
Proof. The matrix of the linearization of (T) at a symmetric equilibrium $(\hat{I}, \hat{V}, \hat{I}, \hat{V})$ reads

$$
\left(\begin{array}{cccc}
-\beta \hat{I}-\alpha & -(1-\sigma) \beta \hat{I} & \alpha & 0 \\
-(\phi+\sigma \beta \hat{V}) & -(\mu+\theta+\phi+\sigma \beta \hat{I})-\alpha & 0 & \alpha \\
\alpha & 0 & -\beta \hat{I}-\alpha & -(1-\sigma) \beta \hat{I} \\
0 & \alpha & -(\phi+\sigma \beta \hat{V}) & -(\mu+\theta+\phi+\sigma \beta \hat{I})-\alpha
\end{array}\right)
$$

where we used the identity $\beta(K-\hat{I}-(1-\sigma) \hat{V})=\mu+\gamma$ from (3.2). With the notation $P=-\beta \hat{I}, Q=-(1-\sigma) \beta \hat{I}, R=-(\phi+\sigma \beta \hat{V})$, and $S=-(\mu+\theta+\phi+\sigma \beta \hat{I})$, we arrive at the characteristic equation

$$
\begin{aligned}
& (P-\alpha-\lambda)^{2}(S-\alpha-\lambda)^{2}-2((P-\alpha-\lambda) Q R(S-\alpha-\lambda)) \\
& \quad+Q^{2} R^{2}-\left((P-\alpha-\lambda)^{2}-2 Q R-(S-\alpha-\lambda)^{2}\right) \alpha^{2}+\alpha^{4}=0 .
\end{aligned}
$$

The characteristic equation factors as

$$
\begin{aligned}
& \left(Q R-(P-\alpha-\lambda)(S-\alpha-\lambda)-\alpha^{2}-\alpha(P+S-2 \alpha-2 \lambda)\right) \\
& \quad\left(Q R-(P-\alpha-\lambda)(S-\alpha-\lambda)-\alpha^{2}+\alpha(P+S-2 \alpha-2 \lambda)\right)=0,
\end{aligned}
$$

so we see that the characteristic roots arise as the solution of one of the following quadratic equations:

$$
\begin{aligned}
\lambda^{2}-(P+S) \lambda+P S-Q R & =0, \\
\lambda^{2}-(P+S-4 \alpha) \lambda+P S-Q R+4 \alpha^{2}-2 \alpha(P+S) & =0 .
\end{aligned}
$$

Solutions of (3.6) depend on $\hat{I}$ and $\hat{V}$; however, by (3.3) the latter can be calculated using the former, so the roots of $(3.6)_{1}$ are obtained as

$$
\begin{aligned}
& \lambda_{1}(\hat{I})=\frac{P+S+\sqrt{(P+S)^{2}-4(P S-Q R)}}{2}, \\
& \lambda_{2}(\hat{I})=\frac{P+S-\sqrt{(P+S)^{2}-4(P S-Q R)}}{2},
\end{aligned}
$$

and the roots of $(3.6)_{2}$ arise as

$$
\begin{aligned}
& \lambda_{3}(\hat{I})=\frac{P+S-4 \alpha+\sqrt{(P+S)^{2}-4(P S-Q R)}}{2}, \\
& \lambda_{4}(\hat{I})=\frac{P+S-4 \alpha-\sqrt{(P+S)^{2}-4(P S-Q R)}}{2} .
\end{aligned}
$$

Using $\beta(K-\hat{I}-(1-\sigma) \hat{V})=\mu+\gamma$, we derive

$$
P S-Q R=\beta \hat{I}(2 \sigma \beta \hat{I}+(\mu+\theta+\sigma \phi)+\sigma(\mu+\gamma)-\sigma \beta K)=\beta \hat{I}(2 A \hat{I}+B),
$$

and it is easy to check that $2 A(\breve{I})^{2}+B>0$ and $2 A(\breve{I})^{1}+B<0$ hold. As $P+S<0$ for both $(\breve{I})^{1}$ and $(\breve{I})^{2}$, and moreover $P S-Q R$ is positive for $(\breve{I})^{2}$ and negative for $(\breve{I})^{1}$, we conclude 
that $\operatorname{Re}\left(\lambda_{1}\left((\breve{I})^{1}\right)\right)>0$ and $\operatorname{Re}\left(\lambda_{1}\left((\breve{I})^{2}\right)\right)<0$. It follows immediately that the equilibrium $(\hat{I}, \hat{V}, \hat{I}, \hat{V})$ with $\hat{I}=(\breve{I})^{1}$ is unstable where it exists. On the other hand, $\alpha>0$ implies that $\operatorname{Re}\left(\lambda_{3}\right)<\operatorname{Re}\left(\lambda_{1}\right)$ and $\operatorname{Re}\left(\lambda_{4}\right)<\operatorname{Re}\left(\lambda_{2}\right)$ hold, so by $\operatorname{Re}\left(\lambda_{2}\right)<\operatorname{Re}\left(\lambda_{1}\right)$ we learn that the real part of $\lambda_{1}$ dominates the real part of the other three roots. It follows that the symmetric equilibrium with $\hat{I}=(\breve{I})^{2}$ is locally asymptotically stable where it exists.

Remark 3. The existence and stability of symmetric equilibria is independent of the mobility parameter $\alpha$.

We note that Proposition 3.7 completes our understanding of the bifurcation behavior of the model $(\mathrm{T})$ in case condition (c) does not hold. The following corollary is given to summarize the findings of Propositions 3.3 and 3.7.

Corollary 3.8. Assume that condition (c) does not hold. Then the system (T) undergoes a forward transcritical bifurcation at $\mathcal{R}_{0}=1$; i.e., the DFE loses its stability as $\mathcal{R}_{0}$ crosses 1 from the left to the right, and the single positive steady state emerging at $\mathcal{R}_{0}=1$ is asymptotically stable.

We recall that the conditions (c) and $\mathcal{R}_{0} \in\left(\mathcal{R}_{c}, 1\right)$ are necessary for the existence of nonsymmetric endemic steady states. The next result characterizes these equilibria.

Proposition 3.9. Assume that condition (c) holds. If $\mathcal{R}_{c}<\mathcal{R}_{0}<1$, then there exist zero, two, four, or six nonsymmetric endemic equilibria (i.e., $\hat{I}_{1} \neq \hat{I}_{2}$ ) in the model $(\mathrm{T})$. For such equilibria, $\left(\hat{I}_{1}, \hat{I}_{2}\right) \in\left((\breve{I})^{1},(\breve{I})^{2}\right) \times\left(0,(\breve{I})^{1}\right)$ if $\hat{I}_{1}>\hat{I}_{2}$, and $\left(\hat{I}_{1}, \hat{I}_{2}\right) \in\left(0,(\breve{I})^{1}\right) \times\left((\breve{I})^{1},(\breve{I})^{2}\right)$ if $\hat{I}_{1}<\hat{I}_{2}$.

Proof. Earlier in this section, the following system was derived for endemic equilibria:

$$
\begin{aligned}
& 0=\frac{1}{\beta \hat{I}_{1} \hat{I}_{2}(\sigma-1)}\left(\alpha^{2}\left(\hat{I}_{1}^{2}-\hat{I}_{2}^{2}\right)+\alpha\left(\hat{I}_{1}-\hat{I}_{2}\right) \hat{I}_{2}\left(\mu+\phi+\beta \hat{I}_{1}(1+\sigma)+\theta\right)\right. \\
& \left.+\hat{I}_{1} \hat{I}_{2} \beta\left(A \hat{I}_{1}^{2}+B \hat{I}_{1}+C\right)\right), \\
& 0=\frac{1}{\beta \hat{I}_{1} \hat{I}_{2}(\sigma-1)}\left(\alpha^{2}\left(\hat{I}_{2}^{2}-\hat{I}_{1}^{2}\right)+\alpha\left(\hat{I}_{2}-\hat{I}_{1}\right) \hat{I}_{1}\left(\mu+\phi+\beta \hat{I}_{2}(1+\sigma)+\theta\right)\right. \\
& \left.+\hat{I}_{1} \hat{I}_{2} \beta\left(A \hat{I}_{2}^{2}+B \hat{I}_{2}+C\right)\right)
\end{aligned}
$$

where

$$
\begin{aligned}
& A=\sigma \beta, \\
& B=(\mu+\theta+\sigma \phi)+\sigma(\mu+\gamma)-\sigma \beta K, \\
& C=\frac{(\mu+\gamma)(\mu+\theta+\phi)}{\beta}-(\mu+\theta+\sigma \phi) K .
\end{aligned}
$$

From $(3.5)_{1}+(3.5)_{2}$ and $(3.5)_{1}-(3.5)_{2}$, and using the fact that $\hat{I}_{1}, \hat{I}_{2} \neq 0$ and $\hat{I}_{1} \neq \hat{I}_{2}$, we obtain

$$
\begin{aligned}
& 0=-\alpha(\mu+\phi+\theta)\left(\hat{I}_{1}-\hat{I}_{2}\right)^{2}+\hat{I}_{1} \hat{I}_{2} \beta\left(A\left(\hat{I}_{1}^{2}+\hat{I}_{2}^{2}\right)+B\left(\hat{I}_{1}+\hat{I}_{2}\right)+2 C\right), \\
& 0=2 \alpha^{2}\left(\hat{I}_{1}+\hat{I}_{2}\right)+\alpha\left(\hat{I}_{1} \hat{I}_{2} \beta(1+\sigma)+\left(\hat{I}_{1}+\hat{I}_{2}\right)(\mu+\phi+\theta)\right)+\hat{I}_{1} \hat{I}_{2} \beta\left(A\left(\hat{I}_{1}+\hat{I}_{2}\right)+B\right),
\end{aligned}
$$

which we reformulate as

$$
\begin{aligned}
& 0=-\alpha(\mu+\phi+\theta)\left(x^{2}-4 y\right)+y \beta\left(A\left(x^{2}-2 y\right)+B x+2 C\right), \\
& 0=2 \alpha^{2} x+\alpha(y \beta(1+\sigma)+x(\mu+\phi+\theta))+y \beta(A x+B),
\end{aligned}
$$


where we let $x=\hat{I}_{1}+\hat{I}_{2}$ and $y=\hat{I}_{1} \hat{I}_{2}$.

Let us assume that $\hat{I}_{1}>\hat{I}_{2}$ at the equilibrium. Then it follows from $(3.5)_{1}$ that $A \hat{I}_{1}^{2}+B \hat{I}_{1}+$ $C<0$, which implies $(\breve{I})^{1}<\hat{I}_{1}<(\breve{I})^{2}$. Similarly, using $(3.5)_{2}$, we derive $A \hat{I}_{2}^{2}+B \hat{I}_{2}+C>0$, so either $\hat{I}_{2}<(\breve{I})^{1}$ or $\hat{I}_{2}>(\breve{I})^{2}$ holds. On the other hand, it follows from $(3.7)_{2}$ and $\alpha>0$, $x, y>0$ that $x=\hat{I}_{1}+\hat{I}_{2}<\frac{-B}{A}$ should be satisfied, which makes $\hat{I}_{2}>(\breve{I})^{2}$ impossible. We conclude that if $\hat{I}_{1}>\hat{I}_{2}$, then $\left(\hat{I}_{1}, \hat{I}_{2}\right) \in\left((\breve{I})^{1},(\breve{I})^{2}\right) \times\left(0,(\breve{I})^{1}\right)$, and similar arguments lead to the result that $\left(\hat{I}_{1}, \hat{I}_{2}\right) \in\left(0,(\breve{I})^{1}\right) \times\left((\breve{I})^{1},(\breve{I})^{2}\right)$ for $\hat{I}_{2}>\hat{I}_{1}$.

We eliminate $y$ using (3.7) 2 and substitute into (3.7) $)_{1}$ to give an equation of $x$ of fourth order. As $(x, y)=(0,0)$ is a solution of $(3.7)$, there are at most three solutions which satisfy $x, y>0$. This implies that for $\hat{I}_{1} \neq \hat{I}_{2}$ the system (3.5) can have zero, two, four, or six solutions, because any $a, b>0$ which satisfy $a+b=x, a b=y$, may serve as $(a, b)=\left(\hat{I}_{1}, \hat{I}_{2}\right)$ and $(b, a)=\left(\hat{I}_{1}, \hat{I}_{2}\right)$.

Unfortunately, due to the complicated coefficients of the fourth order equation, we are unable to determine the exact number of nonsymmetric equilibria. In the next result, a region in the parameter space will be described where six nonsymmetric steady states exist. Furthermore, we illustrate with examples in section 4 that each of the other scenarios can also be realized.

We revisit the results of Propositions 3.1 and 3.2 for the steady states of the model in the case of disconnected regions. If $\alpha=0$, condition (c) holds, and $\mathcal{R}_{0}$ is in the interval $\left(\mathcal{R}_{c}, 1\right)$, then the system has nine equilibria: three of them are symmetric for the two regions and are given as $\hat{I}_{1}=\hat{I}_{2}=0, \hat{I}_{1}=\hat{I}_{2}=(\breve{I})^{1}, \hat{I}_{1}=\hat{I}_{2}=(\breve{I})^{2}$, while $\hat{I}_{1} \neq \hat{I}_{2}$ holds for the remaining six steady states. Now we prove that the model $(\mathrm{T})$ for connected regions has a similar structure of steady states if conditions (c) and $\mathcal{R}_{0} \in\left(\mathcal{R}_{c}, 1\right)$ are satisfied and $\alpha$ is close to 0 . More precisely, in addition to the three symmetric equilibria which exist for any value of $\alpha$ (see Proposition 3.6), the system also possesses six nonsymmetric equilibria if mobility is incorporated with sufficiently small volumes into the model.

Proposition 3.10. Assume that condition (c) holds. If $\mathcal{R}_{c}<\mathcal{R}_{0}<1$, then there is an $\alpha^{*}$ such that the model $(\mathrm{T})$ has six nonsymmetric equilibria for any $\alpha \in\left[0, \alpha^{*}\right)$.

Proof. We let $F=\left(F_{I_{1}}, F_{V_{1}}, F_{I_{2}}, F_{V_{2}}\right)^{T}, F\left(\hat{I}_{1}, \hat{V}_{1}, \hat{I}_{2}, \hat{V}_{2}, \alpha\right): \mathbb{R}^{5} \rightarrow \mathbb{R}^{4}$, and define $F_{I_{1}}$, $F_{V_{1}}, F_{I_{2}}$, and $F_{V_{2}}$ as the right-hand sides of the first, second, third, and fourth equations, respectively, of (3.2). Then the system (3.2) can be reformulated as

$$
\begin{aligned}
& 0=F_{I_{1}}\left(\hat{I}_{1}, \hat{V}_{1}, \hat{I}_{2}, \hat{V}_{2}, \alpha\right), \\
& 0=F_{V_{1}}\left(\hat{I}_{1}, \hat{V}_{1}, \hat{I}_{2}, \hat{V}_{2}, \alpha\right), \\
& 0=F_{I_{2}}\left(\hat{I}_{1}, \hat{V}_{1}, \hat{I}_{2}, \hat{V}_{2}, \alpha\right), \\
& 0=F_{V_{2}}\left(\hat{I}_{1}, \hat{V}_{1}, \hat{I}_{2}, \hat{V}_{2}, \alpha\right) .
\end{aligned}
$$

For $\alpha=0$, we denote the solutions of (3.8) by $E^{0}=\left(\left(\hat{I}_{1}\right)^{0},\left(\hat{V}_{1}\right)^{0},\left(\hat{I}_{2}\right)^{0},\left(\hat{V}_{2}\right)^{0}\right)$. As it was derived in Proposition 3.1, there are nine such solutions and $\left(\hat{I}_{1}\right)^{0},\left(\hat{I}_{2}\right)^{0} \in\left\{0,(\breve{I})^{1},(\breve{I})^{2}\right\}$. 
Using (3.2), we obtain

$$
\begin{aligned}
& \frac{\partial F_{I_{1}}}{\partial \hat{I}_{1}}=\beta\left(K-\hat{I}_{1}-(1-\sigma) \hat{V}_{1}\right)-\beta \hat{I}_{1}-(\mu+\gamma+\alpha), \\
& \frac{\partial F_{I_{1}}}{\partial \hat{V}_{1}}=-\beta(1-\sigma) \hat{I}_{1}, \quad \frac{\partial F_{I_{1}}}{\partial \hat{I}_{2}}=\alpha, \quad \frac{\partial F_{I_{1}}}{\partial \hat{V}_{2}}=0, \\
& \frac{\partial F_{V_{1}}}{\partial \hat{I}_{1}}=-\phi-\sigma \beta \hat{V}_{1}, \\
& \frac{\partial F_{V_{1}}}{\partial \hat{V}_{1}}=-\sigma \beta \hat{I}_{1}-(\mu+\theta+\phi+\alpha), \quad \frac{\partial F_{V_{1}}}{\partial \hat{I}_{2}}=0, \quad \frac{\partial F_{V_{1}}}{\partial \hat{V}_{2}}=\alpha,
\end{aligned}
$$

and similar identities hold for the partial derivatives of $F_{I_{2}}$ and $F_{V_{2}}$. Then the Jacobian $\frac{\partial F}{\partial\left(\hat{I}_{1}, \hat{V}_{1}, \hat{I}_{2}, \hat{V}_{2}\right)^{T}}$, evaluated at $\alpha=0$ and $\left(\hat{I}_{1}, \hat{V}_{1}, \hat{I}_{2}, \hat{V}_{2}\right)=\left(\left(\hat{I}_{1}\right)^{0},\left(\hat{V}_{1}\right)^{0},\left(\hat{I}_{2}\right)^{0},\left(\hat{V}_{2}\right)^{0}\right)$, has the form

$$
\left(\begin{array}{cc}
\mathcal{A}_{1,1} & \mathcal{O} \\
\mathcal{O} & \mathcal{A}_{2,2}
\end{array}\right)
$$

where $\mathcal{O}$ denotes the $2 \times 2$ matrix with zeros and

$$
\begin{aligned}
& \mathcal{A}_{1,1}=\left(\begin{array}{cc}
\beta\left(K-2\left(\hat{I}_{1}\right)^{0}-(1-\sigma)\left(\hat{V}_{1}\right)^{0}\right)-(\mu+\gamma) & -\beta(1-\sigma)\left(\hat{I}_{1}\right)^{0} \\
-\phi-\sigma \beta\left(\hat{V}_{1}\right)^{0} & -\sigma \beta\left(\hat{I}_{1}\right)^{0}-(\mu+\theta+\phi)
\end{array}\right), \\
& \mathcal{A}_{2,2}=\left(\begin{array}{cc}
\beta\left(K-2\left(\hat{I}_{2}\right)^{0}-(1-\sigma)\left(\hat{V}_{2}\right)^{0}\right)-(\mu+\gamma) & -\beta(1-\sigma)\left(\hat{I}_{2}\right)^{0} \\
-\phi-\sigma \beta\left(\hat{V}_{2}\right)^{0} & -\sigma \beta\left(\hat{I}_{2}\right)^{0}-(\mu+\theta+\phi)
\end{array}\right) .
\end{aligned}
$$

If $\left(\hat{I}_{j}\right)^{0}=0$ for any $j \in\{1,2\}$, then we obtain

$$
\begin{aligned}
\operatorname{det}\left(\mathcal{A}_{j, j}\right) & =-\left(\beta\left(K-(1-\sigma)\left(\hat{V}_{1}\right)^{0}\right)-(\mu+\gamma)\right)(\mu+\theta+\phi) \\
& =(\mu+\theta+\phi)(\mu+\gamma)\left(1-\mathcal{R}_{0}\right),
\end{aligned}
$$

since $\left(\hat{I}_{j}\right)^{0}=0$ implies $\left(\hat{V}_{j}\right)^{0}=\bar{V}$. On the other hand, we know that $\beta\left(K-\left(\hat{I}_{j}\right)^{0}-(1-\right.$ $\left.\sigma)\left(\hat{V}_{j}\right)^{0}\right)-(\mu+\gamma)=0$ if $\left(\hat{I}_{j}\right)^{0} \neq 0$; hence in this case we get

$$
\begin{aligned}
\operatorname{det}\left(\mathcal{A}_{j, j}\right) & =\beta\left(\hat{I}_{j}\right)^{0}\left(\sigma \beta\left(\hat{I}_{j}\right)^{0}+(\mu+\theta+\phi)\right)-\left(\phi+\sigma \beta\left(\hat{V}_{j}\right)^{0}\right) \beta(1-\sigma)\left(\hat{I}_{j}\right)^{0} \\
& =\beta\left(\hat{I}_{j}\right)^{0}\left(2 A\left(\hat{I}_{j}\right)^{0}+B\right) .
\end{aligned}
$$

It is clear that $(\mu+\theta+\phi)(\mu+\gamma)\left(1-\mathcal{R}_{0}\right) \neq 0$ for $\mathcal{R}_{0} \in\left(\mathcal{R}_{c}, 1\right)$, and $\left(\hat{I}_{j}\right)^{0} \in\left\{(\breve{I})^{1},(\breve{I})^{2}\right\}$ yields $\beta\left(\hat{I}_{j}\right)^{0}\left(2 A\left(\hat{I}_{j}\right)^{0}+B\right) \neq 0$.

It follows that the Jacobian of the system (3.8) evaluated at $\alpha=0,\left(\hat{I}_{1}, \hat{V}_{1}, \hat{I}_{2}, \hat{V}_{2}\right)=E^{0}$, is nonsingular. Then, by means of the implicit function theorem, there is an interval $\left[0, \alpha^{*}\right)$, an open set $U \in \mathbb{R}^{4}$, and a unique continuously differentiable function $g=\left(g_{I_{1}}, g_{V_{1}}, g_{I_{2}}, g_{V_{2}}\right)$ : $\left[0, \alpha^{*}\right) \rightarrow U$ such that $g(0)=E^{0}$ and $F(g(\alpha), \alpha)=0$ for $\alpha \in\left[0, \alpha^{*}\right)$. This means that $g(\alpha)$ is an equilibrium of the model $(\mathrm{T})$ for connected regions. 
We conclude that if $\alpha$ is close to 0 , then system (3.2) has nine solutions, and each of them is obtained by a unique function of $\alpha$ on an interval $\left[0, \alpha^{*}\right)$. This also means that if the mobility parameter is sufficiently small, then for any equilibrium $E^{0}$ of the disconnected system there exists an equilibrium in the model $(\mathrm{T})$ for connected regions (a solution of the system (3.2)), which is close to $E^{0}$. It remains to show that $g(\alpha) \geq 0$ holds on $\left[0, \alpha^{*}\right)$; that is, the equilibrium of $(\mathrm{T})$ takes nonnegative values and, thus, is biologically meaningful.

Steady states for the vaccinated classes arise by one of the formulas (3.1) and (3.3); thus it is clear that $g_{I_{1}}, g_{I_{2}} \geq 0$ yields $g \geq 0$. We know from Proposition 3.1 that $(\mathrm{T})$ has a DFE for any positive $\alpha$; thus by the uniqueness of $g$, we obtain $g_{I_{1}}(\alpha)=g_{I_{2}}(\alpha)=0$ for $\alpha \in\left[0, \alpha^{*}\right)$ if $\left(\left(\hat{I}_{1}\right)^{0},\left(\hat{I}_{2}\right)^{0}\right)=(0,0)$. Any equilibrium $E^{0}=\left(\left(\hat{I}_{1}\right)^{0},\left(\hat{V}_{1}\right)^{0},\left(\hat{I}_{2}\right)^{0},\left(\hat{V}_{2}\right)^{0}\right)$ with $\left(\hat{I}_{1}\right)^{0},\left(\hat{I}_{2}\right)^{0} \in\left\{(\breve{I})^{1},(\breve{I})^{2}\right\}$ is a positive equilibrium; hence $\alpha^{*}$ can be chosen such that $g(\alpha)>0$ holds for $\alpha<\alpha^{*}$. Next, we consider an equilibrium $E^{0}$ where $\left(\hat{I}_{1}\right)^{0}=0$ and $\left(\hat{I}_{2}\right)^{0}>0$, and we remark that the case when $\left(\hat{I}_{2}\right)^{0}=0,\left(\hat{I}_{1}\right)^{0}>0$ can be treated similarly. We claim that $\frac{\mathrm{d} g_{I_{1}}}{\mathrm{~d} \alpha}(0)>0$ holds. Indeed, using $(3.2)_{1}$ and the definition of $F$, we obtain

$$
\begin{array}{r}
\frac{\mathrm{d}}{\mathrm{d} \alpha} F_{I_{1}}(g(\alpha), \alpha)=0 \\
\beta\left(K-\frac{\mathrm{d} g_{I_{1}}}{\mathrm{~d} \alpha}(\alpha)-(1-\sigma) \frac{\mathrm{d} g_{V_{1}}}{\mathrm{~d} \alpha}(\alpha)\right) g_{I_{1}}(\alpha)+\beta\left(K-g_{I_{1}}(\alpha)-(1-\sigma) g_{V_{1}}(\alpha)\right) \\
\cdot \frac{\mathrm{d} g_{I_{1}}}{\mathrm{~d} \alpha}(\alpha)-(\mu+\gamma+\alpha) \frac{\mathrm{d} g_{I_{1}}}{\mathrm{~d} \alpha}(\alpha)-g_{I_{1}}(\alpha)+\alpha \frac{\mathrm{d} g_{I_{2}}}{\mathrm{~d} \alpha}(\alpha)+g_{I_{2}}(\alpha)=0
\end{array}
$$

which we reformulate and evaluate at $\alpha=0$ to get

$$
\beta\left(K-(1-\sigma) g_{V_{1}}(0)-(\mu+\gamma)\right) \cdot \frac{\mathrm{d} g_{I_{1}}}{\mathrm{~d} \alpha}(0)=-g_{I_{2}}(0)
$$

where we used the fact that $\left(\hat{I}_{1}\right)^{0}=g_{I_{1}}(0)=0$. We note that $g_{V_{1}}(0)=\bar{V}$, and $g_{I_{2}}(0)=$ $\left(\hat{I}_{2}\right)^{0}>0$ holds by assumption. Hence we derive

$$
\begin{aligned}
\frac{\mathrm{d} g_{I_{1}}}{\mathrm{~d} \alpha}(0) & =\frac{-\left(\hat{I}_{2}\right)^{0}}{\beta\left(K-(1-\sigma) \bar{V}_{1}-(\mu+\gamma)\right)}, \\
\frac{\mathrm{d} g_{I_{1}}}{\mathrm{~d} \alpha}(0) & =\frac{\left(\hat{I}_{2}\right)^{0}}{(\mu+\gamma)\left(1-\mathcal{R}_{0}\right)},
\end{aligned}
$$

which is positive if and only if $\mathcal{R}_{0}<1$. By $\frac{\mathrm{d} g_{I_{1}}}{\mathrm{~d} \alpha}(0)>0$ we know that $g_{I_{1}}$ is positive for small $\alpha$. From $\left(\hat{I}_{2}\right)^{0}>0$ it follows that $g_{I_{2}}(\alpha)>0$ holds if $\alpha$ is sufficiently close to 0 ; thus we conclude that $\alpha^{*}$ can be defined such that $g(\alpha)$ is positive for all $\alpha \in\left[0, \alpha^{*}\right)$.

Summarizing, for $\alpha$ sufficiently small, the model (T) has nine steady states. It was shown in Propositions 2.2 and 3.6 that the DFE and two positive symmetric equilibria exist in the model, independent of the value of $\alpha$. The remaining six steady states are thus nonsymmetric equilibria. The proof is thus complete.

We conclude that introducing traveling with small volumes does not change the stability of equilibria. 
Proposition 3.11. In Proposition 3.10, $\alpha^{*}$ can be defined such that the equilibrium $g(\alpha)$ is locally asymptotically stable (unstable) for $\alpha \in\left(0, \alpha^{*}\right)$ whenever $g(0)=E^{0}$ is locally asymptotically stable (unstable).

Proof. The stability of equilibria of the model for disconnected regions has been discussed in Proposition 3.2. If $\left(\hat{I}_{1}\right)^{0},\left(\hat{I}_{2}\right)^{0} \in\left\{0,(\breve{I})^{2}\right\}$, then the steady state $E^{0}=\left(\left(\hat{I}_{1}\right)^{0},\left(\hat{V}_{1}\right)^{0},\left(\hat{I}_{2}\right)^{0},\left(\hat{V}_{2}\right)^{0}\right)$ is locally asymptotically stable, while any equilibrium with the component $(\breve{I})^{1}$ is unstable. If $E^{0}$ is an asymptotically stable equilibrium, then all eigenvalues of $\frac{\partial F}{\partial\left(\hat{I}_{1}, \hat{V}_{1}, \hat{I}_{2}, \hat{V}_{2}\right)^{T}}\left(E^{0}, 0\right)$ have negative real part. Thus, if $\alpha$ is sufficiently small, then it follows by continuity of eigenvalues with respect to parameters that $\frac{\partial F}{\partial\left(\hat{I}_{1}, \hat{V}_{1}, \hat{I}_{2}, \hat{V}_{2}\right)^{T}}(g(\alpha), \alpha)$ has only eigenvalues with negative real part.

We claim that for an unstable equilibrium $E^{0}$ the Jacobian has an eigenvalue with positive real part. If so, then this property is preserved for $g(\alpha)$ as well if $\alpha$ is close to 0 . Indeed, if $\left(\hat{I}_{j}\right)^{0}=(\breve{I})^{1}$ for any $j \in\{1,2\}$, then $\operatorname{det} \mathcal{A}_{j, j}=\beta(\breve{I})^{1}\left(2 A(\breve{I})^{1}+B\right)$ is negative, and by $\beta\left(K-\left(\hat{I}_{j}\right)^{0}-(1-\sigma)\left(\hat{V}_{j}\right)^{0}\right)-(\mu+\gamma)=0$, the first element of the first row in $\mathcal{A}_{j, j}$ simplifies to $-\beta(I)^{1}$, which implies that the matrix has negative trace. It follows immediately that $\mathcal{A}_{j, j}$ has a positive real eigenvalue.

Proposition 3.12. Nonsymmetric equilibria of the model (T) (if they exist) get arbitrarily close to $\left((\breve{I})^{1},(\breve{I})^{1}\right)$ as $\alpha \rightarrow \infty$.

Proof. We show that for every $\epsilon>0$ there exists an $\alpha_{\epsilon}$ such that $\left|\hat{I}_{1}-\hat{I}_{2}\right|<\epsilon$ whenever $\alpha>\alpha_{\epsilon}$.

Let us assume that there is an $\epsilon>0$ such that for any large $\alpha$ there exists a steady state with $\left|\hat{I}_{1}-\hat{I}_{2}\right| \geq \epsilon$. Without loss of generality, we may suppose that $\hat{I}_{1}>\hat{I}_{2}$. By Proposition 3.9 , we know that $\hat{I}_{1}>(\breve{I})^{1}$ and $\hat{I}_{2}>0$. Then, from $(3.5)_{1}$ we derive

$$
\begin{array}{r}
\left(\hat{I}_{1}-\hat{I}_{2}\right)\left(\alpha^{2}\left(\hat{I}_{1}+\hat{I}_{2}\right)+\alpha \hat{I}_{2}\left(\mu+\phi+\beta \hat{I}_{1}(1+\sigma)+\theta\right)\right)=-\hat{I}_{1} \hat{I}_{2} \beta\left(A \hat{I}_{1}^{2}+B \hat{I}_{1}+C\right), \\
\epsilon \cdot \alpha^{2}\left(\hat{I}_{1}+\hat{I}_{2}\right) \leq-\hat{I}_{1} \hat{I}_{2} \beta\left(A \hat{I}_{1}^{2}+B \hat{I}_{1}+C\right) .
\end{array}
$$

The left-hand side of the last inequality is positive and unbounded, and it follows from the positivity of $A$ that the right-hand side is bounded from above. We get by contradiction that for large $\alpha$ the components for the infected classes of a nonsymmetric equilibrium are arbitrarily close to each other. We also know that $\left(\hat{I}_{1}, \hat{I}_{2}\right) \in\left(\left((\breve{I})^{1},(\breve{I})^{2}\right) \times\left(0,(\breve{I})^{1}\right)\right) \cup\left(\left(0,(\breve{I})^{1}\right) \times\right.$ $\left((\breve{I})^{1},(\breve{I})^{2}\right)$ ) (see Proposition 3.9), which yields the statement.

We observe that the existence and stability of symmetric equilibria was fully characterized in this section. It was also noted in Remark 3 that these fixed points are independent of $\alpha$. However, our results on nonsymmetric steady states are only partial; as a matter of fact, we are going to show that these equilibria are sensitive to $\alpha$ and other model parameters in the sense that various interesting bifurcations can occur in the model. We conclude this section with a summary of our findings in the case when (c) holds.

Corollary 3.13. Assume that condition (c) holds. Then system (T) exhibits a saddlenode bifurcation at $\mathcal{R}_{0}=\mathcal{R}_{c}$, where two symmetric equilibria emerge. Depending on the value of $\alpha$ and other model parameters, there can exist six nonsymmetric steady states on the interval $\mathcal{R}_{0} \in\left(\mathcal{R}_{c}, 1\right)$. In such a case, the stable DFE coexists with three stable and five unstable nontrivial equilibria. There are no nonsymmetric steady states for $\mathcal{R}_{0} \geq 1$. The system undergoes a backward transcritical bifurcation at $\mathcal{R}_{0}=1$, where the unstable 
symmetric equilibrium bifurcates into the DFE, and DFE loses its stability. The symmetric endemic steady state which is stable on $\mathcal{R}_{0} \in\left(\mathcal{R}_{c}, 1\right)$ exists for $\mathcal{R}_{0} \geq 1$ and is stable.

4. Numerical simulations for the model $(T)$. We conducted numerical simulations in order to illustrate the rich bifurcation behavior in the model (T). In section 3, the dynamics was fully described in the case when condition (c) for multiple positive equilibria does not hold. Moreover, we characterized steady states and their stability when (c) is satisfied and either $\mathcal{R}_{0}<\mathcal{R}_{c}$ or $\mathcal{R}_{0}>1$. Under the condition (c), there are two symmetric positive equilibria in addition to the DFE in the interval $\left(\mathcal{R}_{c}, 1\right)$, and they coexist with six nonsymmetric steady states if the travel rate between the regions is sufficiently small. However, as will be illustrated in this section, nonsymmetric equilibria are sensitive to $\alpha$; i.e., they disappear for larger travel volumes.

For all simulations in this section, we fix model parameters as $K=100, \mu=0.1, \gamma=12$, $\theta=0.5, \sigma=0.2, \phi=16$. It is easy to check that $\mathcal{R}_{c} \approx 0.9224$ and that condition (c) is satisfied. For four different values of $\mathcal{R}_{0}$ in the interval $\left(\mathcal{R}_{c}, 1\right)$, we illustrate in Figure 2 how the positive steady states are destroyed by the increase in the travel rate between the regions. With all the other parameters fixed, we let $\alpha$ increase from 0 through small volumes to larger rates, and we depict the equilibria with colors changing from green $(\alpha=0)$ through blue and violet to red $(\alpha=1)$. Varying $\alpha$ has no effect on the steady states in the diagonal (see also Proposition 3.6). On the other hand, nonsymmetric fixed points exist only for small travel volumes. More precisely, there are six such equilibria for $\alpha=0$ (see Proposition 3.1 for disconnected regions), which continue to exist for small volumes of travel, and they move along some curves as a function of the travel rate.

Figure 2 demonstrates that, for larger values of $\alpha$, nonsymmetric equilibria vanish in two saddle-node bifurcation points and a pitchfork-like bifurcation point of the unstable positive symmetric equilibrium. It is interesting to note the differences in the bifurcation diagrams at different values of $\mathcal{R}_{0}$. In case of Figures $2(\mathrm{a})$ and (b) when $\mathcal{R}_{0}$ is only slightly greater than $\mathcal{R}_{c}$, two fully endemic equilibria move towards the diagonal, and two other pairs of steady states - those which are partially endemic for $\alpha=0$ - collide in saddle-node bifurcations. This behavior is different from that experienced for larger values of $\mathcal{R}_{0}$, depicted in Figures 2(c) and (d). In these diagrams, the fully endemic fixed points undergo saddle-node bifurcations, and there are two partially endemic equilibria of the disconnected system, which continue to exist with traveling until they bifurcate into the unstable positive steady state at the diagonal. We recall the result of Theorem 3.9 to remark that nonsymmetric equilibria and their bifurcations are contained in $(\hat{I})^{1},(\hat{I})^{2} \in\left((\breve{I})^{1},(\breve{I})^{2}\right) \times\left(0,(\breve{I})^{1}\right) \cup\left(0,(\breve{I})^{1}\right) \times\left((\breve{I})^{1},(\breve{I})^{2}\right)$.

Figures 3 and 4 give some more insight into the dynamical behavior in the model. We noted two different bifurcation structures in Figure 2. Now we pick two parameter settings which result in different bifurcations and investigate the long-time behavior of solutions and stability of steady states for various values of $\alpha$. Dots represent equilibria, and solutions converging to a particular steady state are depicted with the same color. It was shown in Proposition 3.11 that, for small volumes of travel, each of the nine steady states of the model ( $\mathrm{T}$ ) is close to a steady state of disconnected regions $(\alpha=0)$ and has the same stability. Figures 3(a) and 4(a) further support this result. On the other hand, equilibria move and sometimes disappear as $\alpha$ increases; hence the attractors of certain solutions change. We illustrate with Figures 3(b), 


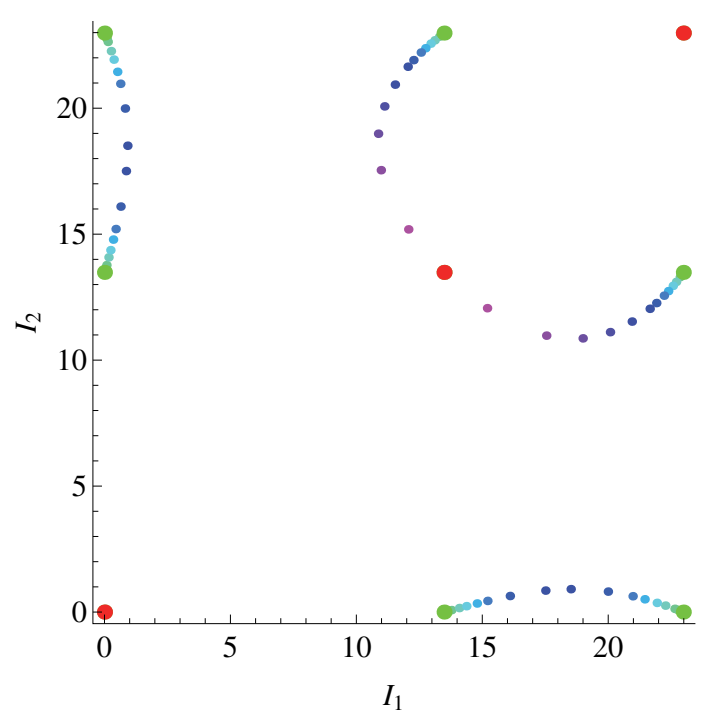

(a) $\mathcal{R}_{0} \approx 0.9261(\beta=0.4895)$.

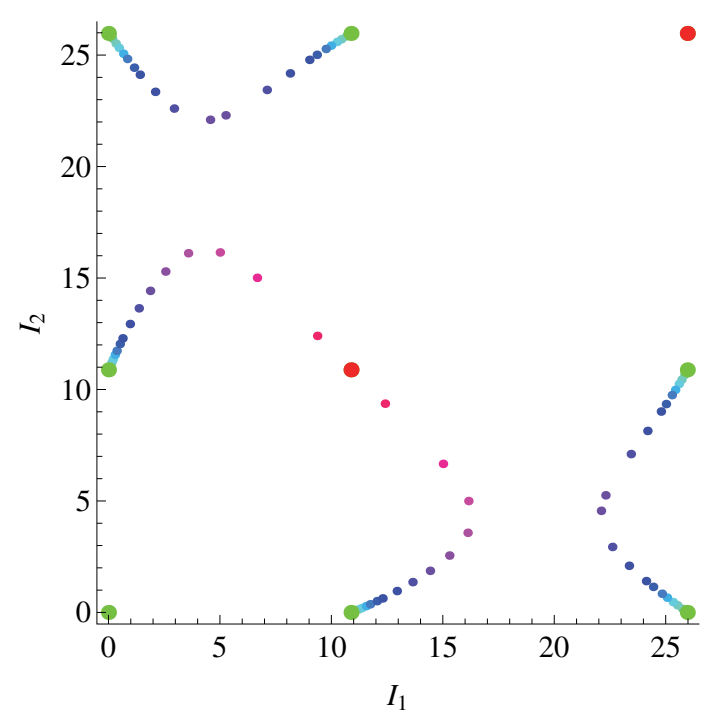

(c) $\mathcal{R}_{0} \approx 0.9317(\beta=0.4925)$.

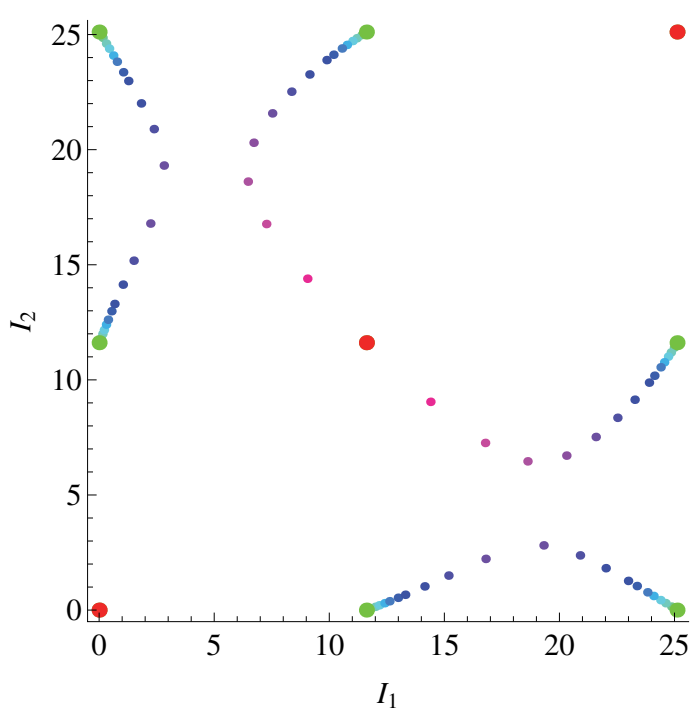

(b) $\mathcal{R}_{0} \approx 0.9299(\beta=0.4915)$.

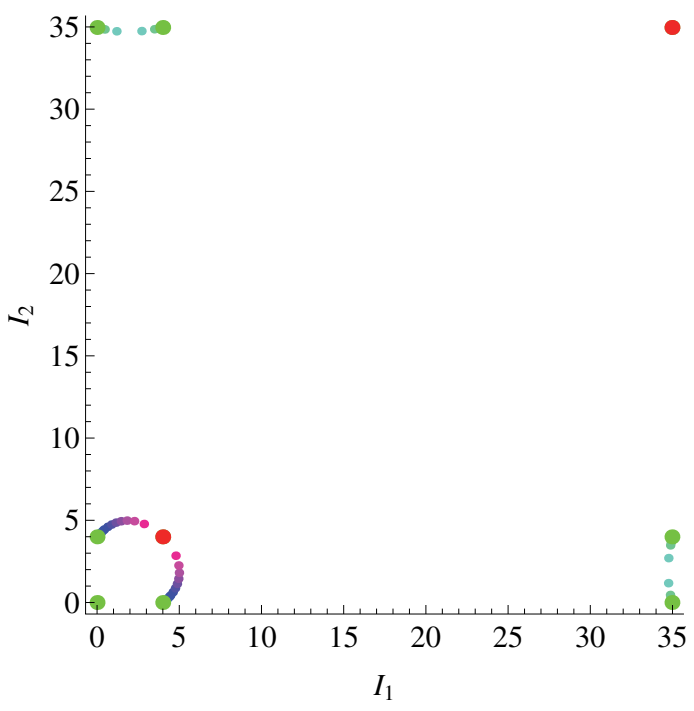

(d) $\mathcal{R}_{0} \approx 0.9639(\beta=0.5095)$.

Figure 2. Equilibria of the model $(\mathrm{T})$ when $\mathcal{R}_{c}<\mathcal{R}_{0}<1$ is satisfied and the travel rate $\alpha$ varies between 0 and 1. The fixed points in the diagonal exist for all travel volumes, and for $\alpha=1$ (red) there are no other equilibria. In case when the regions are disconnected (i.e., $\alpha=0$ ) there also exist six nonsymmetric steady states (green). Each such fixed point moves along a unique continuous function as $\alpha$ increases (color changes from green through blue and violet to red). For larger travel volumes, nonsymmetric equilibria disappear as they collide (saddle-node bifurcation) or bifurcate into a fixed point in the diagonal (pitchfork-like bifurcation, with three unstable branches). Parameters were set as $K=100, \mu=0.1, \gamma=12, \theta=0.5, \sigma=0.2, \phi=16$. This makes $\mathcal{R}_{c} \approx 0.9224$, and condition (c) for multiple nontrivial equilibria is also satisfied. 


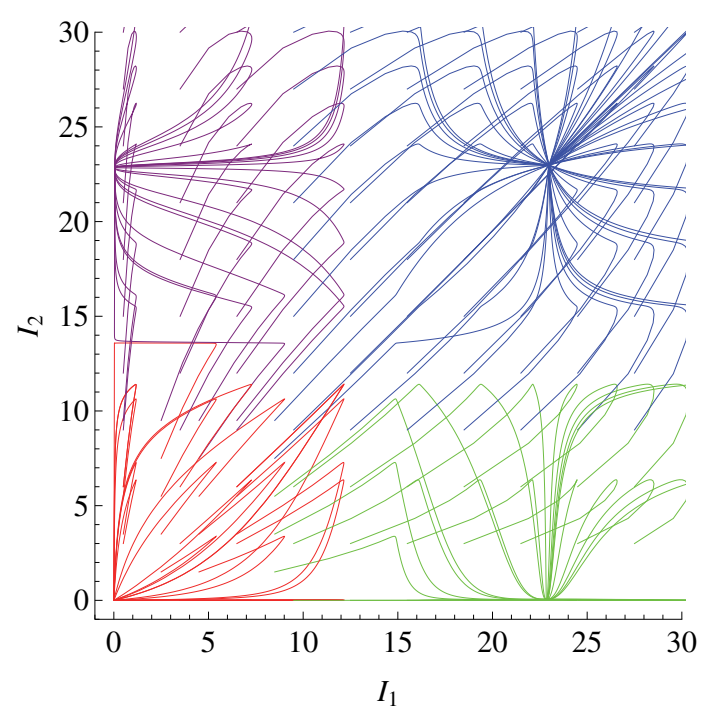

(a) $\alpha=0.002$.

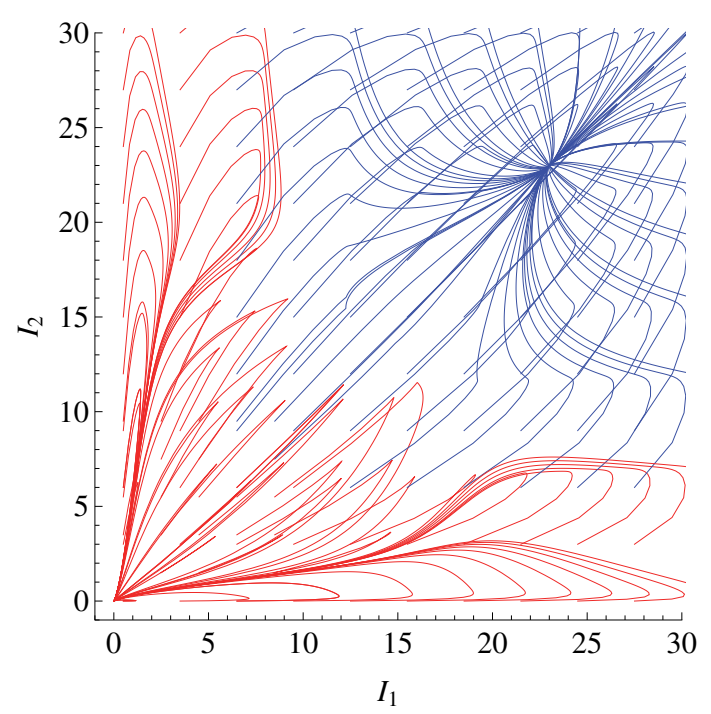

(c) $\alpha=0.1$.

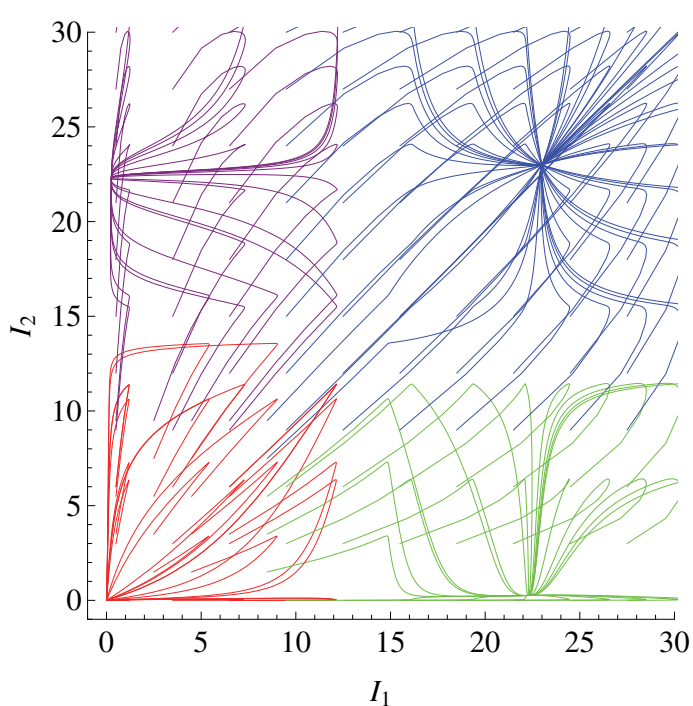

(b) $\alpha=0.01$.

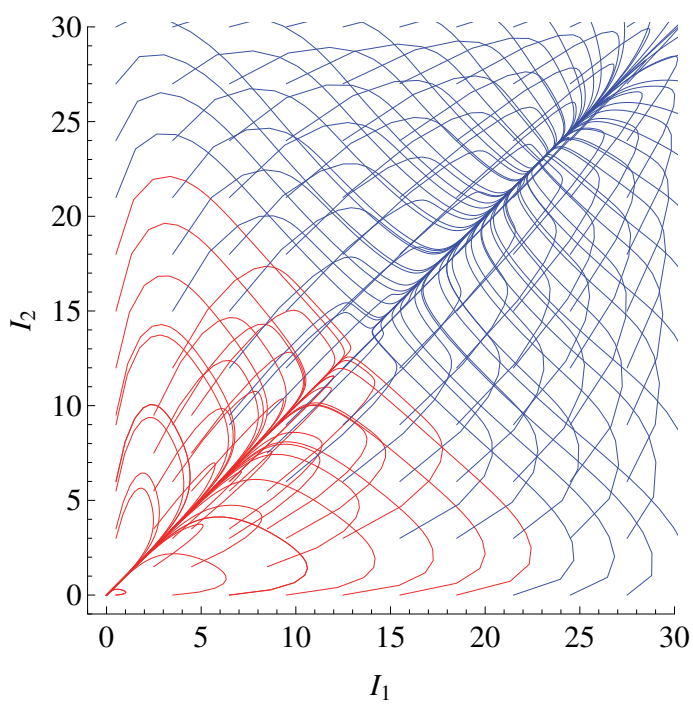

(d) $\alpha=1$.

Figure 3. Solutions of the model (T) for four values of $\alpha$, when the bifurcations of equilibria are as illustrated in Figure 2(a). Solutions converging to an equilibrium point are indicated with the same color. There are several attractors when the travel volume is small; however, for larger values of $\alpha$, all solutions seem to converge to the stable equilibria in the diagonal. Parameters were set as $K=100, \mu=0.1, \gamma=12, \theta=0.5$, $\sigma=0.2, \phi=16, \beta=0.4895$. This makes $\mathcal{R}_{0} \approx 0.9261$ and $\mathcal{R}_{c} \approx 0.9224$, and condition (c) for multiple nontrivial equilibria is also satisfied.

(c), (d) and Figures 4(b), (c), (d) that the difference in the dynamics for $\mathcal{R}_{0}=0.926068$ and $\mathcal{R}_{0}=0.931744$, detected in the bifurcation phenomena (Figures $2(\mathrm{a})$ and (c)), also manifests itself in the long-time behavior of solutions.

Figure 5 shows equilibria (blue dots) of the model (T) for four different values of the travel 


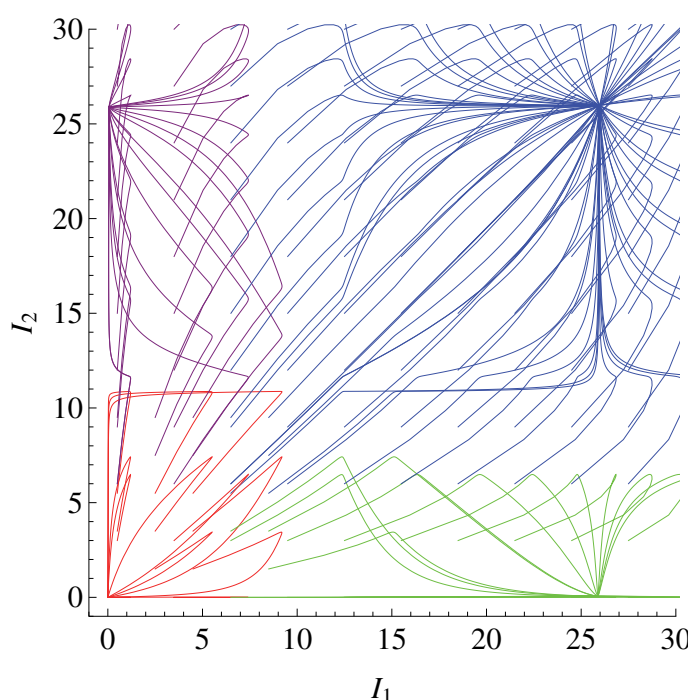

(a) $\alpha=0.002$.

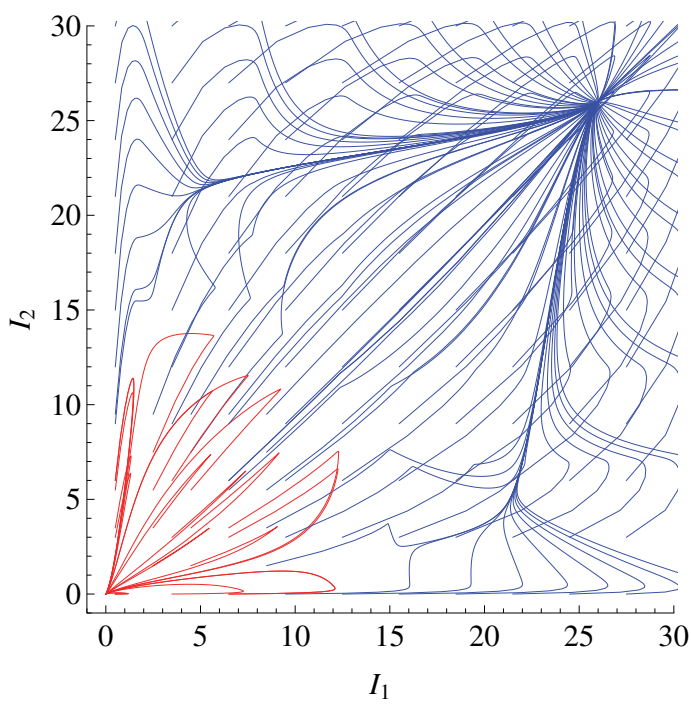

(c) $\alpha=0.1$.

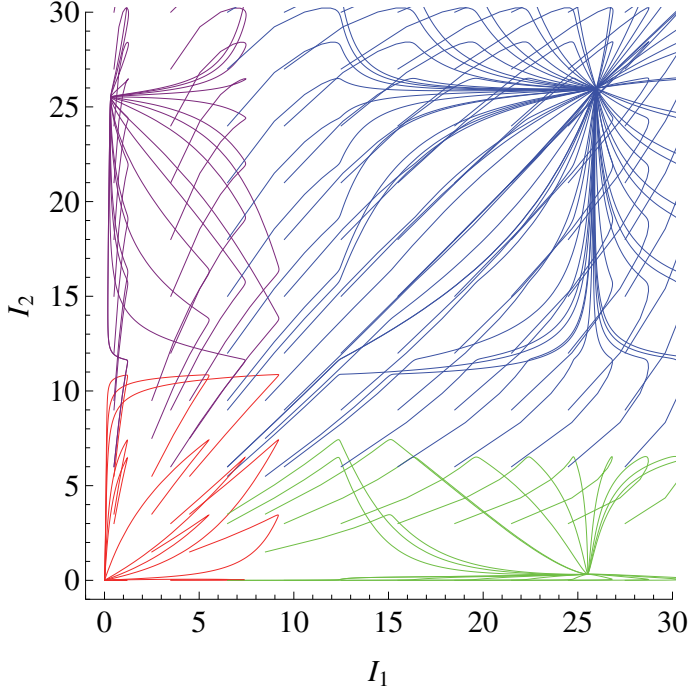

(b) $\alpha=0.01$

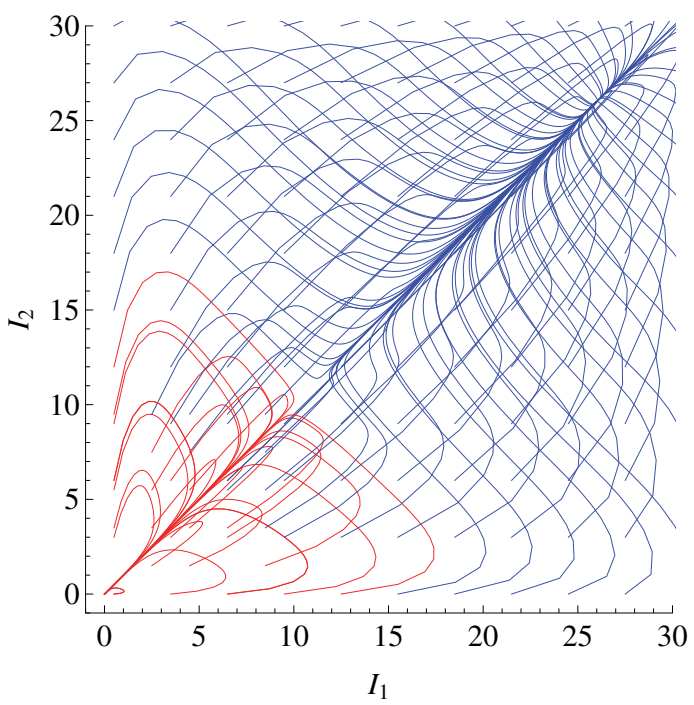

(d) $\alpha=1$.

Figure 4. Solutions of the model (T) for four values of $\alpha$ when the bifurcations of equilibria are as illustrated in Figure 2(c). Solutions converging to an equilibrium point are indicated with the same color. There are several attractors when the travel volume is small; however, for larger values of $\alpha$, all solutions seem to converge to the stable equilibria in the diagonal. Parameters were set as $K=100, \mu=0.1, \gamma=12, \theta=0.5, \sigma=0.2, \phi=16$, $\beta=0.4925$. This makes $\mathcal{R}_{0} \approx 0.9317$ and $\mathcal{R}_{c} \approx 0.9224$, and condition (c) for multiple nontrivial equilibria is also satisfied.

rate $\alpha$. We also depict the steady states of the model $\left(\mathrm{T}_{0}\right)$ for disconnected regions (green dots). For $\mathcal{R}_{0}<\mathcal{R}_{c}$, only the DFE exists. When the regions are disconnected, two symmetric endemic and six nonsymmetric endemic equilibria bifurcate as $\mathcal{R}_{0}$ crosses $\mathcal{R}_{c}$, and only three of these fixed points continue to exist when $\mathcal{R}_{0}$ exceeds 1 (see Proposition 3.2). Incorporating 


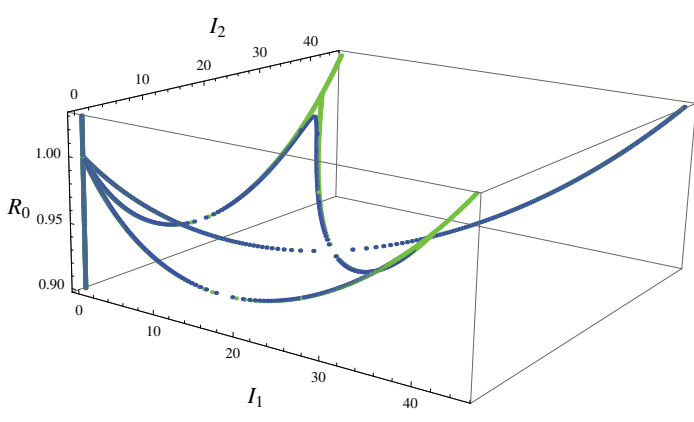

(a) $\alpha=0.002$.

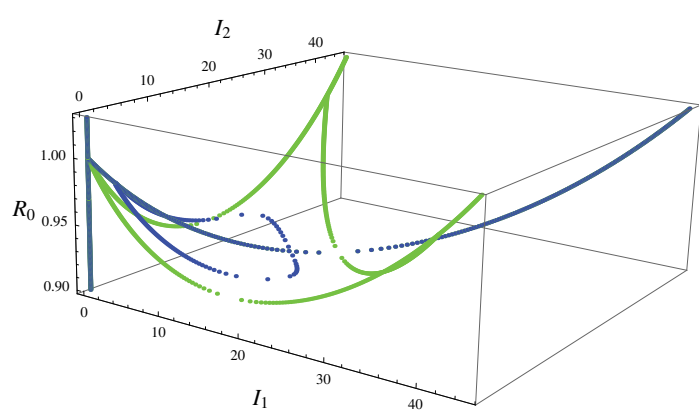

(c) $\alpha=0.1$.

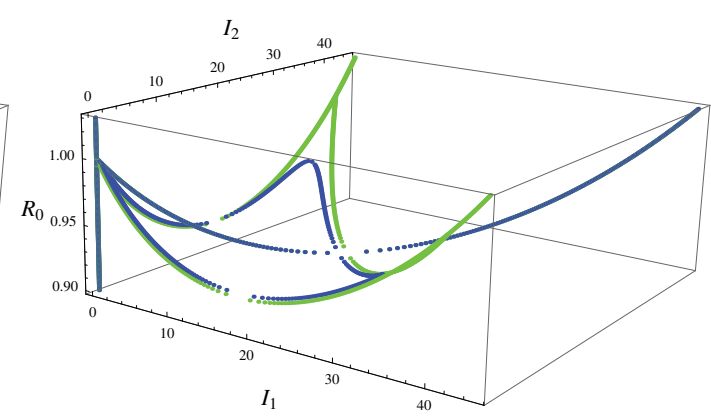

(b) $\alpha=0.02$

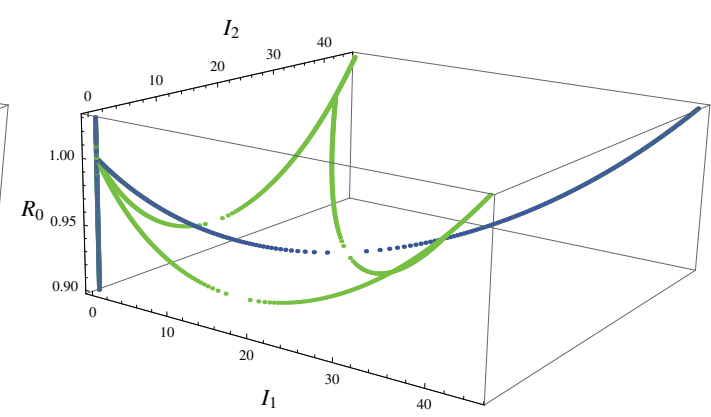

(d) $\alpha=1$.

Figure 5. Equilibria of the model $(\mathrm{T})$ in the case of isolated regions ( $\alpha=0$, green) and connected regions $\left(\alpha>0\right.$, blue) as $\mathcal{R}_{0}$ varies between 0.9 and 1.03. Blue dots in the diagonal overlap green dots. There are no endemic steady states if $\mathcal{R}_{0}<\mathcal{R}_{c}$, and for $\mathcal{R}_{0}>1$ only a single, symmetric positive equilibrium exists if $\alpha>0$. The four figures illustrate that, depending on the values of $\alpha$ and $\mathcal{R}_{0}$, the model may have zero, two, four, or six nonsymmetric fixed points when $\mathcal{R}_{0} \in\left(\mathcal{R}_{c}, 1\right)$. Parameters were set as $K=100, \mu=0.1, \gamma=12$, $\theta=0.5, \sigma=0.2, \phi=16$. This makes $\mathcal{R}_{c} \approx 0.9224$, and condition (c) for multiple nontrivial equilibria is also satisfied.

traveling between the regions into the model does not have any impact on symmetric equilibria; that is, blue dots in the diagonal overlap fixed points of the disconnected system. However, the structure of nonsymmetric steady states strongly depends on the value of the travel parameter. For instance, incorporating traveling at a small rate (e.g., $\alpha=1 / 500$ in Figure 5(a)) modifies equilibrium values only slightly in comparison to the case of disconnected regions, but for $\alpha=1$ (Figure $5(\mathrm{~d})$ ) the system of connected regions has no nonsymmetric steady states. The statement of Proposition 3.6 about the possible numbers of nonsymmetric equilibria is also demonstrated with these plots.

For a fixed travel rate $\alpha=0.05$, we show another figure for the steady states of the system $(\mathrm{T})$. In the three- and two-dimensional plots of Figure 6 , we indicate with different colors how the structure of equilibria changes when we vary $\mathcal{R}_{0}$ in $\left(\mathcal{R}_{c}, 1\right)$. From the orange color corresponding to $\mathcal{R}_{0}=\mathcal{R}_{c}$, the color evolves through yellow, green, blue, and violet into red as $\mathcal{R}_{0}$ increases to 1 . We learned from Proposition 3.6 that two symmetric steady states (one unstable and one stable) are born at $\mathcal{R}_{0}=\mathcal{R}_{c}$; the large orange dot in the plots indicates this saddle-node bifurcation point. From rigorous numerical computations (to be 


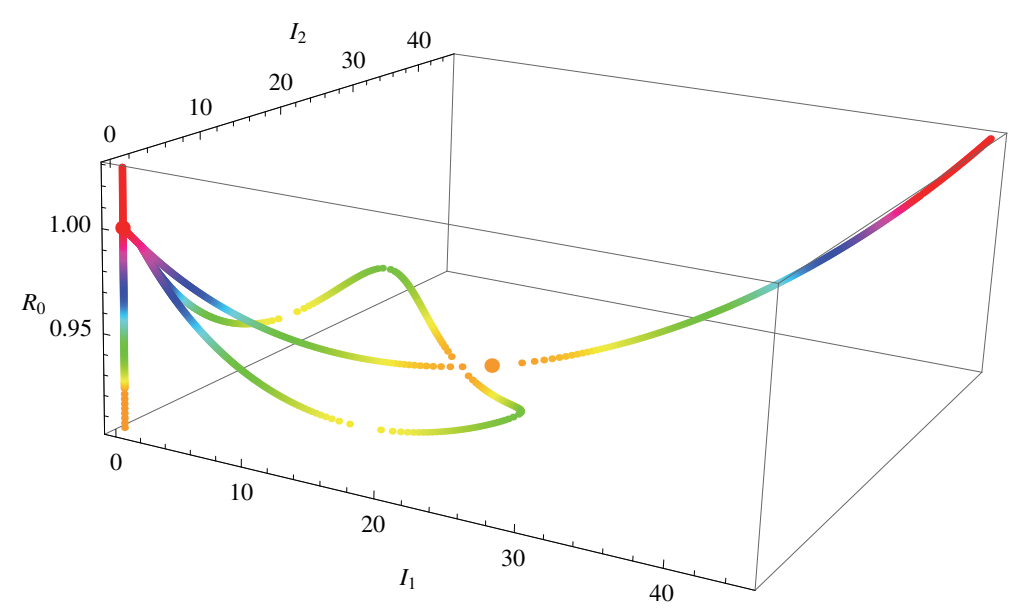

(a) Three-dimensional plot.

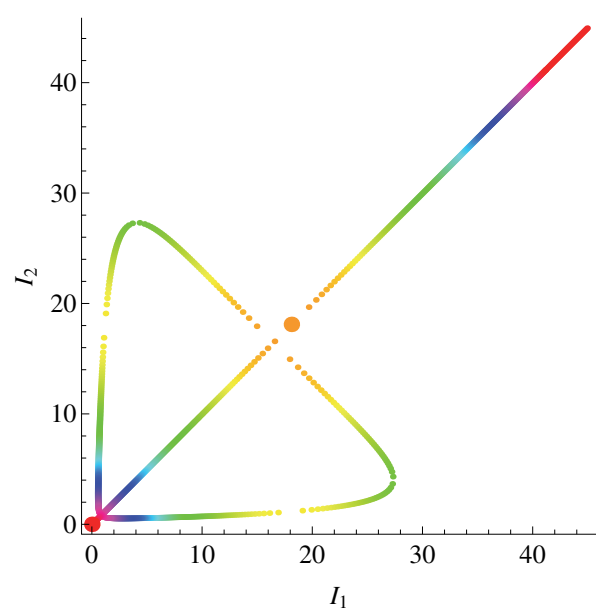

(b) Two-dimensional plot.

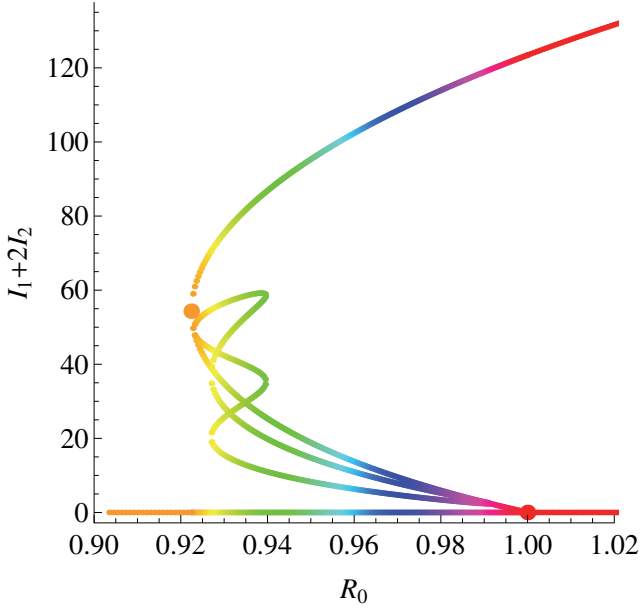

(c) Bifurcation diagram.

Figure 6. Equilibria of the model (T) for $\alpha=0.05$ as $\mathcal{R}_{0}$ varies between 0.9 and 1.02 . Color changes from orange through yellow, green, blue, and violet to red as $\mathcal{R}_{0}$ increases from $\mathcal{R}_{c}$ to 1 . Steady states for $\mathcal{R}_{0}<\mathcal{R}_{c}$ and for $\mathcal{R}_{0}>1$ are depicted by orange and red, respectively. From a saddle-node bifurcation point, one stable and one unstable symmetric positive equilibrium bifurcate at $\mathcal{R}_{0}=\mathcal{R}_{c}$ (large orange dot), which coexist with the DFE for all values of $\mathcal{R}_{0} \in\left(\mathcal{R}_{c}, 1\right)$. As $\mathcal{R}_{0}$ exceeds $\mathcal{R}_{c}$, two nonsymmetric unstable fixed points emerge from the unstable symmetric equilibrium (orange); then two saddle-node bifurcations occur (yellow) to give rise to four other nonsymmetric steady states, pairwise stable-unstable. As $\mathcal{R}_{0}$ further increases, four nonsymmetric fixed points cease to exist as they pairwise collide in two subcritical saddle-node bifurcations (green); then finally all nonsymmetric steady states disappear when two unstable fixed points bifurcate into the unstable symmetric positive equilibrium (pink). At $\mathcal{R}_{0}=1$, the dynamics undergoes a backward transcritical bifurcation (large red dot). Parameters were set as $K=100, \mu=0.1, \gamma=12, \theta=0.5, \sigma=0.2, \phi=16$. This makes $\mathcal{R}_{c} \approx 0.9224$, and condition (c) for multiple nontrivial equilibria is also satisfied.

discussed below) we derive that two unstable equilibria emerge from the unstable symmetric fixed point when $\mathcal{R}_{0}$ is slightly greater than $\mathcal{R}_{c}$ (orange), and the existence of two saddlenode bifurcation points (yellow) are also confirmed, from which four nonsymmetric steady 
states bifurcate (pairwise unstable-stable). As $\mathcal{R}_{0}$ increases, four nonsymmetric fixed points disappear (green, two subcritical saddle-node bifurcations); then the unstable equilibrium in the diagonal absorbs the remaining two nonsymmetric steady states (pink, two unstable fixed points join into the symmetric equilibrium). Again, by the results of Proposition 3.6, the system undergoes a backward transcritical bifurcation at $\mathcal{R}_{0}=1$, which is indicated by a large red dot in the figure.

In the model $\left(\mathrm{T}_{0}\right)$ for disconnected regions, a triple saddle-node bifurcation occurs at $\mathcal{R}_{0}=\mathcal{R}_{c}$ (see Proposition 3.2). We conjecture that incorporating travel between the regions results in the split-up of this bifurcation point, so that one stable and three unstable fixed points emerge in the model $(\mathrm{T})$ when $\mathcal{R}_{0}$ is close to $\mathcal{R}_{c}$. Similarly, the system $\left(\mathrm{T}_{0}\right)$ undergoes a triple backward bifurcation of the DFE at $\mathcal{R}_{0}=1$, which is deformed for positive travel rates: in the model $(\mathrm{T})$ for connected regions, two unstable fixed points join into another unstable steady state as $\mathcal{R}_{0}$ approaches 1 , and then at $\mathcal{R}_{0}=1$ this unstable equilibrium bifurcates into the DFE in a (simple) backward bifurcation.

5. Rigorous set-oriented numerical computations for the model (T). In order to complement the analytical calculations, and to confirm selected results of the numerical simulations, we conducted rigorous set-oriented numerical analysis of global dynamics encountered in the system $(\mathrm{T})$. We applied the framework that was developed in [1] (see also [6]) for discrete-time semidynamical systems. This approach has already been applied successfully to some other systems (see, e.g., $[21,33])$ and in each case provided valuable information. Since the computational method was introduced in [1] for continuous maps only, its extension to ODEs was developed by means of applying this method to a time- $t$ map of the flow. The correctness of this approach is justified in section 5.2; the reader is referred to [13] for an in-depth discussion of some technical aspects of the adaptation of the method to ODEs.

The core idea behind the computational method introduced in [1] is to decompose the dynamics at each selection of parameters into a collection of isolating neighborhoods that encompass all the chain recurrent dynamics in the phase space (e.g., fixed points or periodic solutions) and connecting orbits between them (and also orbits that run away in forward or backward time). The isolating neighborhoods are constructed in such a way that connecting orbits between them define a strict partial order, like in a gradient system. This construction is carried out for small subsets of parameters. Then adjacent subsets of parameters for which equivalent decompositions have been found are joined together to form larger classes. As a result, regions of parameters which yield equivalent global dynamics are identified, and schematic description is computed for the different types of global dynamics.

We begin with a description of this method. Then we explain how this method is applied to the system (T). Finally, we describe the results obtained and discuss how they relate to the findings shown in the previous sections. The software used for the computations, as well as the results, are all made publicly available at the website [32].

5.1. Description of the numerical method. Let $\mathbb{T}=\mathbb{Z}$ or $\mathbb{T}=\mathbb{R}$. Let $\varphi: \mathbb{R}^{n} \times \mathbb{T} \ni$ $(x, t) \mapsto \varphi_{t}(x) \in \mathbb{R}^{n}$ be a dynamical system; that is, for all $x, t_{1}, t_{2}$ we have $\varphi(x, 0)=x$ and $\varphi\left(\varphi\left(x, t_{1}\right), t_{2}\right)=\varphi\left(x, t_{1}+t_{2}\right)$. If $\mathbb{T}=\mathbb{R}$, then we call $\varphi$ a continuous-time dynamical system (or a flow for short), and if $\mathbb{T}=\mathbb{Z}$, then we call it a discrete-time dynamical system.

A set $S$ is called an invariant set with respect to $\varphi$ if $\varphi(S, \mathbb{T})=S$. The invariant part 
of a set $N$, denoted $\operatorname{Inv} N$, is the largest, in terms of inclusion, invariant set contained in $N$. The set $N$ is called an isolating neighborhood if $N$ is compact and $\operatorname{Inv} N \subset \operatorname{int} N$, where $\operatorname{int} N$ denotes the interior of $N . S$ is called an isolated invariant set if $S=\operatorname{Inv} N$ for some isolating neighborhood $N$.

A Morse decomposition (see [8]) of an isolated invariant set $X$ (note that $X$ may be the entire phase space) with respect to $\varphi$ is a finite collection of disjoint isolated invariant subsets $S_{1}, \ldots, S_{q}$ of $X$ (called Morse sets) with a strict partial ordering $\prec$ on the index set $\{1, \ldots, q\}$ such that for every $x \in X \backslash\left(S_{1} \cup \cdots \cup S_{q}\right)$ and for every orbit $\left\{\gamma_{t}\right\}_{t \in \mathbb{T}}$ such that $\gamma_{0}=x$ there exist indices $i \prec j$ such that $\gamma_{t} \rightarrow S_{i}$ as $t \rightarrow \infty$ and $\gamma_{t} \rightarrow S_{j}$ as $t \rightarrow-\infty$.

A rectangular set is a product of compact intervals. Given a rectangular set $R=\left[a_{1}, a_{1}+\right.$ $\left.\delta_{1}\right] \times \cdots \times\left[a_{n}, a_{n}+\delta_{n}\right] \subset \mathbb{R}^{n}$ and integer numbers $s_{1}, \ldots, s_{n}>0$, we call the following set an $s_{1} \times \cdots \times s_{n}$ uniform rectangular grid in $R$ :

$$
\mathcal{G}_{s_{1}, \ldots, s_{n}}(R):=\left\{\prod_{i=1}^{n}\left[a_{i}+\frac{j_{i}}{s_{i}} \delta_{i}, a_{i}+\frac{j_{i}+1}{s_{i}} \delta_{i}\right]: j_{i} \in\left\{0, \ldots, s_{i}-1\right\}, i \in\{1, \ldots, n\}\right\} .
$$

The individual boxes in the grid are indexed by the $n$-tuples $\left(j_{1}, \ldots, j_{n}\right)$.

Now consider an $m$-parameter family of flows on $\mathbb{R}^{n}$ :

$$
\varphi: \mathbb{R}^{n} \times \mathbb{R}^{m} \times \mathbb{R} \ni(x, p, t) \mapsto \varphi_{t}^{p}(x) \in \mathbb{R}^{n} .
$$

Let $B \subset \mathbb{R}^{n}$ and $P \subset \mathbb{R}^{m}$ be rectangular sets.

Let $\tau>0$, and consider the $m$-parameter discrete-time dynamical system $\varphi_{\tau}$ obtained by restriction of $\varphi$ to $\mathbb{R}^{n} \times \mathbb{R}^{m} \times \tau \mathbb{Z}$. Let $d_{1}, \ldots, d_{n}$ and $s_{1}, \ldots, s_{m}$ be positive integers. For each parameter box $\hat{p} \subset P$ in the $s_{1} \times \cdots \times s_{m}$ uniform rectangular grid in $P$, and for each box $b$ in the $d_{1} \times \cdots \times d_{n}$ uniform rectangular grid in $B$, we use the CAPD software library [7] to compute a rigorous outer estimate for $\varphi(b, \hat{p}, \tau)$. In this way, we apply the computational method introduced in [1] to $\varphi_{\tau}$. In particular, the family of sets $N_{1}, \ldots, N_{q} \subset B$ is constructed with some strict partial ordering $\prec$ on $\{1, \ldots, q\}$ such that, for each $p \in \hat{p}$, each set $N_{i}, i=1, \ldots, q$, is an isolating neighborhood in $B$, and whenever a possibility of the existence of an orbit from $N_{i}$ to $N_{j}$ is detected, the relation $N_{j} \prec N_{i}$ is set to hold true. The family $\left\{S_{i}:=\operatorname{Inv} N_{i}: i=1, \ldots, q\right\}$ forms a Morse decomposition of $\operatorname{Inv} B$ with respect to $\varphi_{\tau}^{p}$ with the ordering $\prec$, where $\varphi_{\tau}^{p}=\varphi_{\tau}(\cdot, p, \cdot)$ indicates the dynamical system $\varphi_{\tau}$ with the parameter fixed to $p$. The sets $N_{i}$ are constructed as unions of closed boxes with respect to the $d_{1} \times \cdots \times d_{n}$ uniform rectangular grid in $B$. The collection $N_{1}, \ldots, N_{q}$ is called a numerical Morse decomposition, and the isolating neighborhoods $N_{1}, \ldots, N_{q}$ are called numerical Morse sets. Note that if $N_{i}$ touches the boundary of $B$ for some $i$, then it is not known whether $N_{i}$ is an isolating neighborhood in the entire phase space $X$, so caution should be taken when drawing conclusions from such a construction.

A numerical Morse decomposition can be schematically depicted as a directed graph whose vertices correspond to the Morse sets and whose edges indicate possible connecting orbits between them. In order to simplify such a representation, one can plot the transitive reduction of this graph, as was done in the presentation of the results at [32].

The Conley index, introduced by Conley [8] for flows, and generalized, e.g., by Mrozek [27] and Szymczak [35] to discrete semidynamical systems induced by continuous maps, is a 
topological invariant that provides information about isolated invariant sets. Its homological version is algorithmically computable (to certain extent) from an isolating neighborhood and an outer estimate of the map, like those computed by the method being described. This index takes into account the exit set of an isolating neighborhood $N$, that is, the part of the forward image of $N$ that sticks out of $N$, and thus reflects the stability of what $N$ contains.

Knowledge of the Conley index of an isolating neighborhood $N$ allows us to draw conclusions on the invariant part of $N$. In particular, if the index of $N$ is nontrivial, then $\operatorname{Inv} N \neq \emptyset$. The index can also be used to prove the existence of periodic orbits or more complicated dynamics.

For the purpose of this paper, the Conley index and the relation of the forward image of $N$ are used in order to classify each computed isolating neighborhood $N$ on the basis of stability. We say that an isolating neighborhood $N$ is attracting if the forward image of $N$ is entirely contained in $N$. One can prove that then $N$ contains a local attractor, which justifies this terminology. Otherwise, if the forward image of $N$ is not fully contained in $N$, we say that $N$ is unstable. If $N$ has the Conley index of a hyperbolic fixed point with $d$-dimensional unstable manifold, then we say that $N$ is of the type of the corresponding point. For a typical system, it is likely that $N$ indeed contains an equilibrium of the expected stability, butsince the Conley index is a purely topological tool and does not provide information about derivatives - the dynamics in $N$ may turn out to be much more complicated than seen from outside (that is, from the perspective of the isolating neighborhood). If $N \subset \mathbb{R}^{n}$ is of the type of a fixed point with $n$-dimensional unstable manifold, then we say that $N$ is repelling.

Since detailed introduction to the Conley index is beyond the scope of this paper and requires certain knowledge of algebraic topology, we refer the reader to [8, 27, 35] for more details on the Conley index, and to $[17,25,34]$ and references therein for discussion of some technical aspects of the method for the computation of this index implemented in the software used in this paper.

5.2. Justification of the method for ODEs. In order to apply the method for automatic analysis of global dynamics to a continuous-time dynamical system (a flow) induced by an ODE, it is natural to consider a time- $\tau$ map for some fixed $\tau>0$ and to conduct the computations for the discrete-time dynamical system induced by this map. The following theorem justifies this approach, as it shows that the results obtained from the computations conducted for the time- $\tau$ map are also valid for the flow.

Theorem 5.1. Let $\varphi$ be a flow on $\mathbb{R}^{n}$. Let $\tau>0$. Let $B \subset \mathbb{R}^{n}$ be an isolating neighborhood with respect to $\varphi_{\tau}$. Assume that $N_{1}, \ldots, N_{k} \subset B$ are some isolating neighborhoods for $\varphi_{\tau}$, with pairwise disjoint interiors. Assume that $\mathbb{M}:=\left\{M_{i}:=\operatorname{InvN}_{i} \mid i=1, \ldots, k\right\}$ is a Morse decomposition of $\operatorname{Inv}\left(B, \varphi_{\tau}\right)$ with respect to $\varphi_{\tau}$. Then $N_{1}, \ldots, N_{k}$ are isolating neighborhoods for $\varphi$, and $\mathbb{M}$ is a Morse decomposition of $\operatorname{Inv}(B, \varphi)$ with respect to $\varphi$. Moreover, if there exists a connecting orbit in $B$ for $\varphi$ between some of the Morse sets, then there exists a connecting orbit in $B$ for $\varphi_{\tau}$ between the same Morse sets.

Note that in this theorem there is no one-to-one correspondence between objects computed for the time- $\tau$ map and the flow; it only speaks to one direction of implication. In particular, an isolating neighborhood for the flow need not be an isolating neighborhood for the time$\tau$ map. Moreover, there might exist a connecting orbit for $\varphi_{\tau}$ in $B$ with no corresponding 
connecting orbit for $\varphi$ in $B$.

Before proving this theorem, let us recall a few relevant results.

Proposition 5.2 (see [28, Theorem 1] for an even more general version). For a flow $\varphi$ on $\mathbb{R}^{n}$, the following conditions are equivalent:

(1) $S$ is an isolated invariant set with respect to $\varphi$,

(2) $S$ is an isolated invariant set with respect to $\varphi_{\tau}$ for all $\tau>0$,

(3) $S$ is an isolated invariant set with respect to $\varphi_{\tau}$ for some $\tau>0$.

Proposition 5.3 (see [28, Corollary]). The cohomological Conley index of an isolated invariant set of a flow $\varphi$ coincides with the corresponding index with respect to the discrete dynamical system $\varphi_{\tau}$ for any $\tau>0$.

Proposition 5.4 (see [30, Lemma 6] or [28, proof of Theorem 1, last paragraph, p. 309]). Let $\tau>0$. If $N$ is an isolating neighborhood with respect to a time-discretization $\varphi_{\tau}$ of a flow $\varphi$, then $N$ is an isolating neighborhood with respect to the flow $\varphi$ and $\operatorname{Inv}\left(N, \varphi_{\tau}\right)=\operatorname{Inv}(N, \varphi)$.

Proof of Theorem 5.1. By Proposition 5.4, $B$ is an isolating neighborhood for the flow $\varphi$ and $\operatorname{Inv}(B, \varphi)=\operatorname{Inv}\left(B, \varphi_{\tau}\right)$. Also all $N_{i}$ are isolating neighborhoods for the flow $\varphi$, and $M_{i}=\operatorname{Inv}\left(N_{i}, \varphi\right)$. Either by Proposition 5.2 or by the fact that $M_{i}=\operatorname{Inv}\left(N_{i}, \varphi\right)$ and thus $M_{i} \subset \operatorname{int} N_{i}$, all the $M_{i}$ are isolated invariant sets with respect to the flow $\varphi$. Define $N:=$ $\bigcup_{i=1}^{k} N_{i}$. Since $\operatorname{Inv}\left(B \backslash N, \varphi_{\tau}\right)=\emptyset$, it follows from Proposition 5.4 that also $\operatorname{Inv}(B \backslash N, \varphi)=\emptyset$.

Consider a trajectory with respect to the flow $\varphi$ that connects $M_{i}$ and $M_{j}$ in $B$ for some $i, j \in\{1, \ldots, k\}$. (Since $B$ is an isolating neighborhood, this trajectory is actually contained in $\operatorname{int} B$.) Then a time- $\tau$ discretization of this trajectory is an orbit with respect to $\varphi_{\tau}$ that connects $M_{i}$ and $M_{j}$. As a consequence, all the relations induced by the flow on $\mathbb{M}$ must also exist in the Morse ordering of $\mathbb{M}$ with respect to $\varphi_{\tau}$. It follows that the flow-induced relations on $\mathbb{M}$ can be extended to a partial order on $\mathbb{M}$ by transitivity. Therefore, $\mathbb{M}$ is a Morse decomposition of $\operatorname{Inv}(B, \varphi)$ with respect to the flow $\varphi$.

Regarding some technicalities, we remark that by Proposition 5.3 the Conley indices with respect to the flow $\varphi$ can be instantly obtained from those computed when considering $\varphi_{\tau}$. Unfortunately, the Conley index cannot be computed in certain cases, which we discuss further in section 5.4. Moreover, we may not know whether the chosen set $B$ is indeed an isolating neighborhood (and, in fact, in our computations it is not). Therefore, one can only be sure that $N_{i} \subset B$ is an isolating neighborhood if actually $N_{i} \subset \operatorname{int} B$. In fact, this problem is one of the reasons for why the Conley index could not be computed for the neighborhoods of some equilibria, mainly for the origin.

Choosing an optimal value of $\tau>0$ is not a trivial task. In fact, in our approach, we use a heuristic method which chooses a supposedly good $\tau>0$ by trial and error: $\tau$ is initially chosen quite arbitrarily, and then is increased if possible or decreased if it yields too high overestimates in the computation of outer enclosures of the images of grid elements by $\varphi_{\tau}$.

5.3. Application of the method to the model ( $\mathrm{T})$. The input to the rigorous set-oriented numerical method applied to the system $(\mathrm{T})$ consists of the data listed below. All the real numbers below should be understood as their double-precision binary floating-point approximations.

(I1) The four-dimensional ODE defined by $(\mathrm{T})$, in which we fix the following parameters: $K:=100, \mu:=0.1, \gamma:=12, \theta:=0.5, \sigma:=0.2, \phi:=16$. 
(I2) The ranges of the varying parameters $\alpha \in[0,0.2]$ and $\beta \in[0.48,0.53]$; that is, $P:=$ $[0,0.2] \times[0.48,0.53]$.

(I3) The phase space bounding box that contains all the asymptotic dynamics of our interest: $B:=[0,100]^{4}$. In our case, this box contains the entire feasible phase space $X$ for the model; see (2.5).

(I4) The resolutions in the parameter space and in the phase space. We subdivide the parameter space $P$ uniformly into $80 \times 200$ boxes, and the phase space $B$ into $2^{9}=512$ boxes in each direction. The resolutions were set high enough so that the nine equilibria discussed earlier are clearly visible (see Propositions 3.6 and 3.10), and the bifurcations shown in Figure 2 can also be followed.

In addition to this initial data, there are also several technical parameters, such as a suggestion for $\tau$, which we do not discuss here. All of these technicalities can be easily found in the software available at [32].

The computations can be run at a computer cluster in a convenient way, using a flexible dynamic parallelization scheme introduced in [31], which is built into the software.

The output of the computations consists of the following information:

(O1) Classes of parameters for which the qualitative global dynamics is equivalent. These classes are given as subsets of $P$, built of the boxes into which $P$ was subdivided.

(O2) For each parameter box, selected information about the computed numerical Morse decomposition: the number of sets, their sizes (in terms of the number of boxes), their Conley indices (whenever it was possible to compute them), and information on the detected possible connecting orbits.

(O3) A projection onto $I_{1}$ and $I_{2}$ of the phase space portrait of the sets of which the numerical Morse decomposition is composed. (This form of output is normally optional, as the amount of the data can be overwhelming.)

Illustration and discussion of the obtained results of the computations are gathered in the next section.

5.4. Results of the computations and discussion. Figure 7 shows the continuation diagram computed for the parameters under consideration. Each continuation class consisting of more than one element is indicated in some solid color, with colors repeated for small classes that are at some distance from each other. Thirteen major classes are given labels (a), (b), $\ldots,(\mathrm{m})$ and are briefly discussed below. The reader is invited to explore the details at the interactive presentation provided at the website [32].

In Figure 8, projections of the phase space portraits of the computed numerical Morse decompositions to the coordinates $I_{1}$ and $I_{2}$ are shown for a sample parameter box taken from each of the first 12 classes. In order to save space, the phase space portrait is not shown for class $(\mathrm{m})$, because it is very simple: A single isolating neighborhood of the origin was found there, and it is an attractor.

The constructed isolating neighborhoods capture all the chain recurrent dynamics found in $B$. In particular, these sets contain all the equilibria. If some equilibria are close to each other, then they often cannot be separated at the resolution at which the computations were carried out, and then they share a neighborhood. Moreover, if the hyperbolicity (contraction, expansion) in the vicinity of an equilibrium is strong, then the constructed neighborhood is 


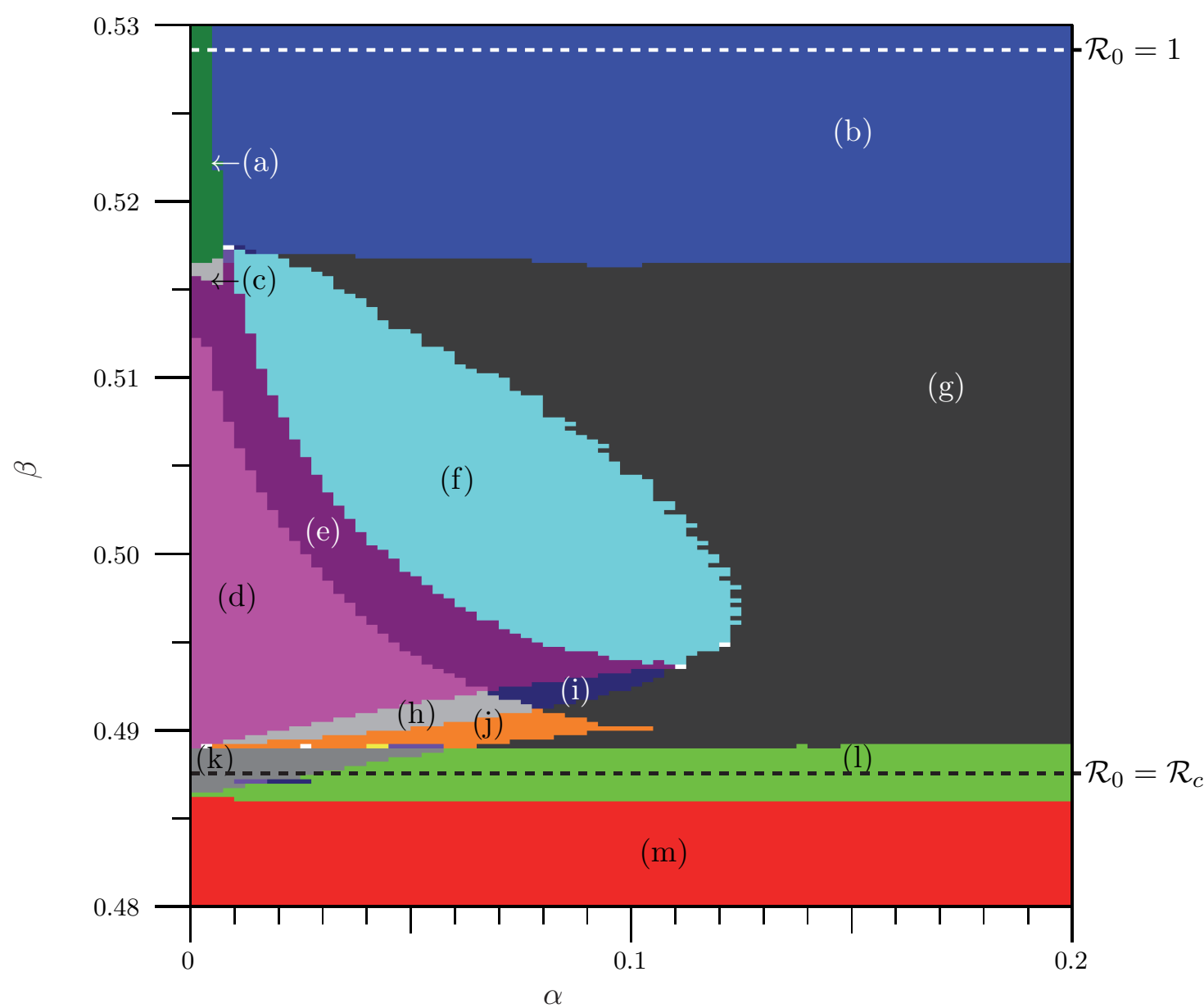

Figure 7. Continuation diagram for the system (T) computed as described in section 5.3. Classes of parameters for which the dynamics is equivalent are indicated in solid colors. Major continuation classes are labeled, and the global dynamics found in each of them is discussed in the text and shown in Figures 8 and 9.

typically very small. However, if the dynamics slows down, then the constructed isolating neighborhoods tend to be larger. Since these are outer estimates for components of chain recurrent dynamics, there are combinatorial trajectories that traverse the entire neighborhood (transitivity). In particular, in an actual system that is modeled, one might expect to see trajectories that run within the entire region of an isolating neighborhood yet correspond to the steady state. Noise and small perturbations pull these trajectories away from the steady state, and its weak stability allows for that.

In Figure 9 we provide schematic description of global dynamics found for the cases shown in Figure 8. In each of the directed graphs, the nodes correspond to the isolating neighborhoods that encompass all the chain recurrent dynamics in $B$, and the paths in these graphs indicate possible connecting orbits in the system. At each node, the consecutive number of the corresponding isolating neighborhood is given. These neighborhoods are numbered in the order in which they were actually computed by the software. The size of each neighborhood in terms of the number of boxes is also provided. The homological Conley index is shown 

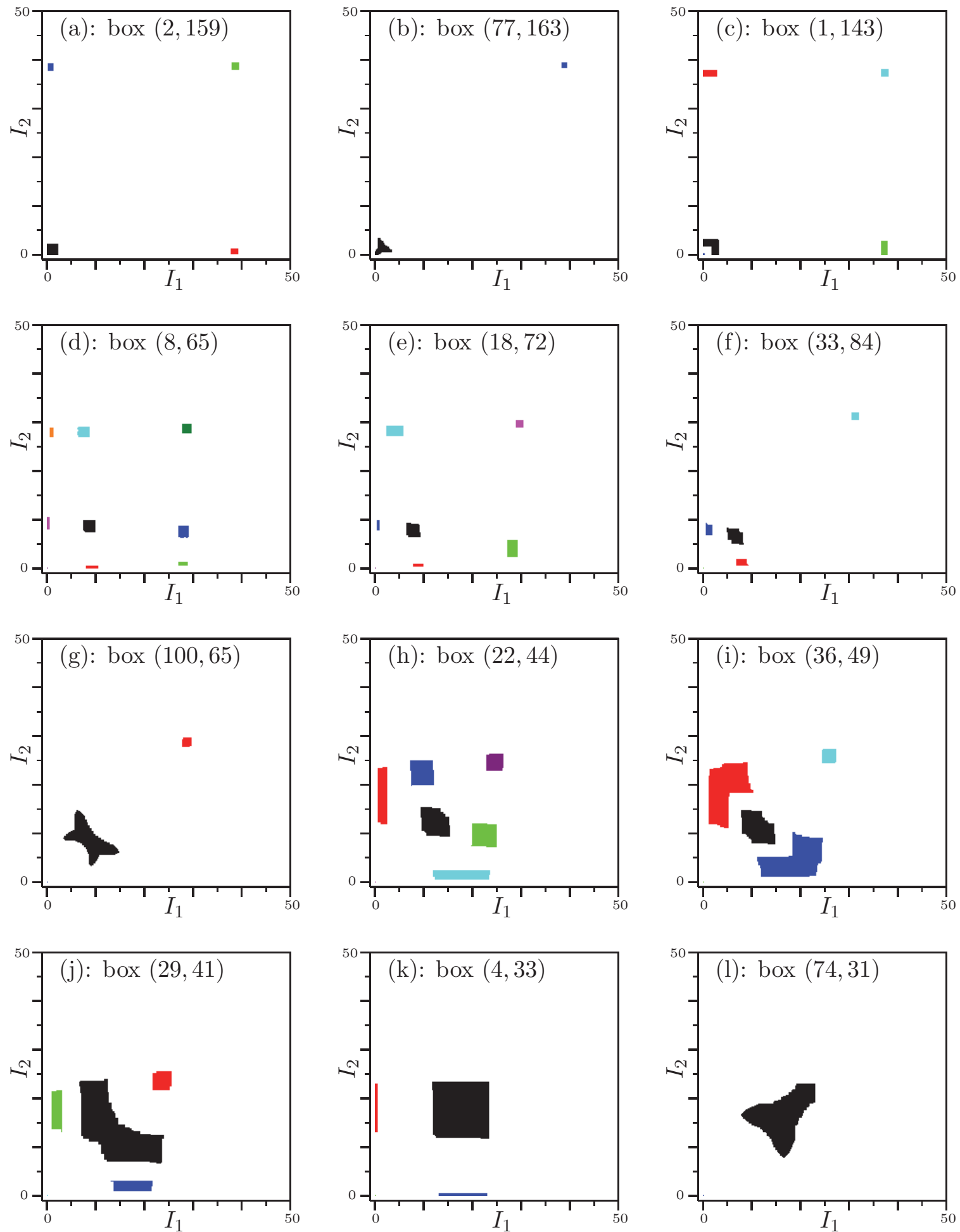

Figure 8. Sample phase space portraits of numerical Morse decompositions computed for selected parameter boxes chosen from the thirteen major continuation classes labeled in Figure 7 . In class (m), which is not shown here, a single isolating neighborhood containing the origin was constructed. Note that the size of the neighborhood of the origin (which is an equilibrium for all the parameters) is so small in all the cases except for (a) and (b) that it is barely visible in the pictures. 


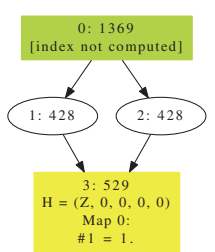

(a)

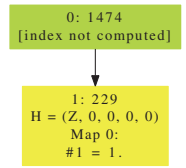

(b)

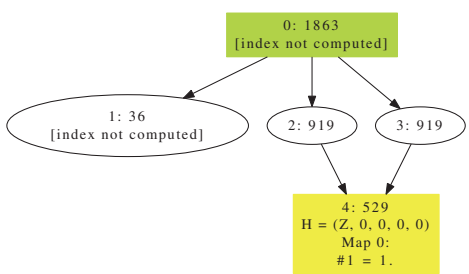

(c)

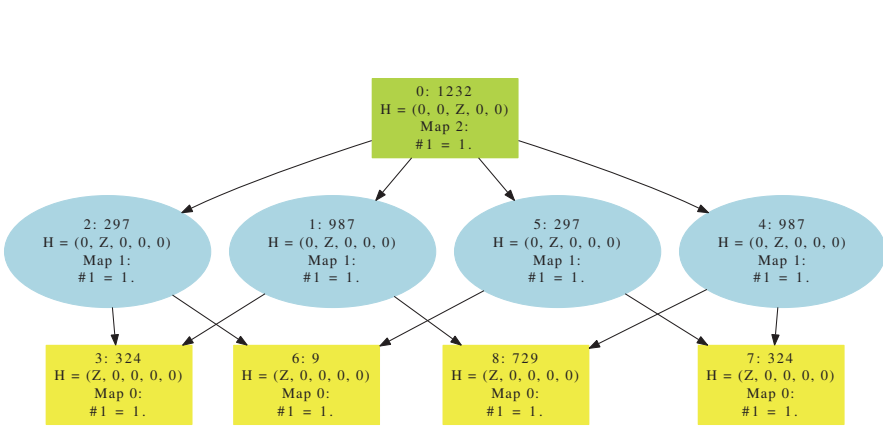

(d)

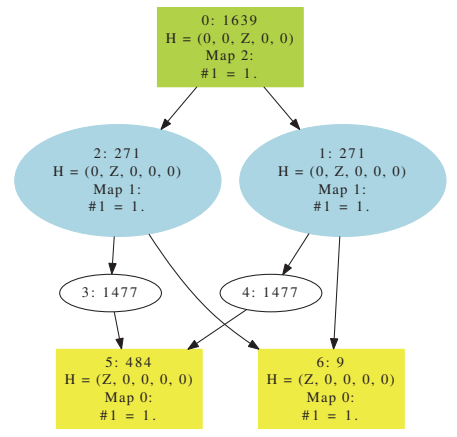

(e)

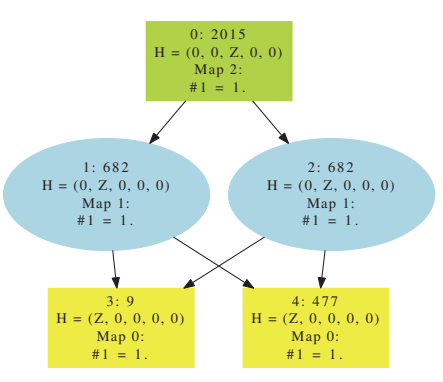

(f)

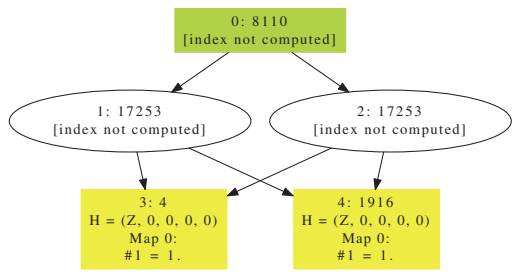

(i)

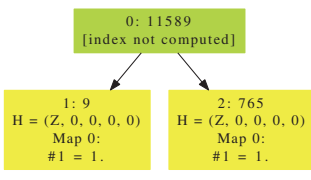

(g)

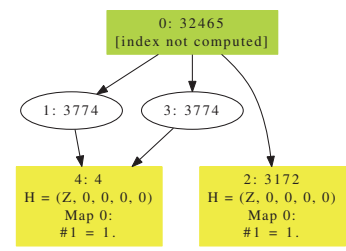

(j)

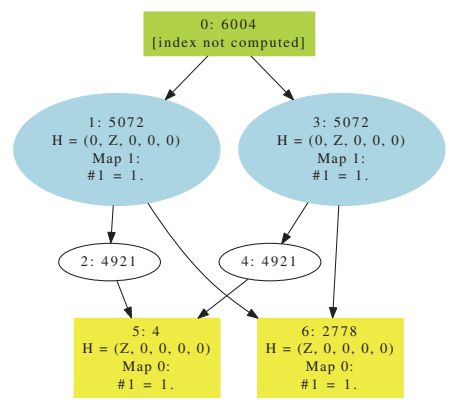

(h)

Figure 9. Conley-Morse diagrams for the sample phase space portraits of numerical Morse decompositions illustrated in Figure 8. The information at each vertex of each graph consists of the consecutive number of the Morse set, its size in terms of the number of boxes, and the Conley index (none in the case of the trivial index). The consecutive numbers of the numerical Morse sets correspond to the following colors in the phase space portraits shown in Figure 8: black, blue, red, green, cyan, pink, violet, orange, dark green. 
whenever the software was able to compute it and the index is nontrivial. Most typical reasons for failures include proximity of the boundary of $B$, and thus lack of isolation, or excessive size of the constructed index pair (over 10,000 boxes), which would compromise the efficiency of the computations, and thus the computation of the index was skipped in such cases. If the index was computed, then the relative homology of the index pair is given, and the maps at the nontrivial homology levels are indicated on homology generators in a compact way by providing the image of each generator (represented by its consecutive number, starting with 1) as a linear combination of the generators. For example, "Map 2: \#1 = 1" means that at homology level 2, the image of homology generator 1 equals this generator (that is, the map is the identity). No information about the index, like in the two nodes of the graph in class (a), indicates the trivial index.

The information shown in Figure 9 complements Figure 8, and the two should be analyzed together. The nine isolating neighborhoods found in class (d) correspond to the nine equilibria whose existence was shown in Proposition 3.10. Indeed, the Conley indices in Figure 9(d) agree with their types of stability: There are four attractors (numbers 3, 6, 7, and 8), four sets of the type of a saddle critical point with one-dimensional unstable manifold, and one set of the type of a critical point with two-dimensional unstable manifold. With the increase in the parameter $\alpha$, there are different possible scenarios of bifurcations that yield to class $(\mathrm{g})$, in which the chain recurrent dynamics is restricted to three isolating neighborhoods along the diagonal. Traversing through classes (e) and (f) corresponds to the scenario depicted in Figure 2(c)-(d), and traversing through classes $(\mathrm{h})$ and $(\mathrm{j})$ corresponds to the scenario depicted in Figure 2(a)-(b). Note that the configuration encountered in class (i) corresponds to an intermediate situation in between these two sequences of bifurcations.

As predicted by the theory, in class (b) - that is, for high values of $\beta$, which correspond to high values of $\mathcal{R}_{0}$ - the chain recurrent dynamics is restricted to two isolating neighborhoods located along the diagonal: one including the DFE, whose Conley index could not be computed due to problems with isolation (the DFE is unstable), and the other corresponding to the endemic equilibrium, which is asymptotically stable. Similarly, in class (m) - that is, for low values of $\beta$, which correspond to low values of $\mathcal{R}_{0}$ - there is exactly one chain recurrent set in $B$, which corresponds to the DFE, which is asymptotically stable in that case.

Several numerical artifacts, which are in fact features of the method that was applied, can easily be noticed in the results of the computations. In particular, several numerical Morse sets with trivial indices were found in classes (a), (c), (e), (h), (i), (j), (k). In all the cases, these sets split into pairs of neighborhoods of the type of an attracting fixed point and a saddle with one-dimensional unstable manifold. These isolating neighborhoods are thus indicators of a slow-down in the dynamics, which is related to the bifurcations taking place for nearby parameter values. It may also be the case that such neighborhoods in fact contain the two equilibria, but they cannot be separated due to their proximity.

It is worth noting that while the line $\mathcal{R}_{0}=\mathcal{R}_{c}$ indeed corresponds to the location at which the Morse decompositions undergo a change that suggests the bifurcation, this is not the case with the line $\mathcal{R}_{0}=1$, which is located well above the border between classes (b) and (g). The reason for this discrepancy is that the different equilibria that participate in the bifurcation get very close together for parameters below $\mathcal{R}_{0}=1$, and thus they are not separated into distinct isolating neighborhoods at the resolution at which the computations were carried out. 
This is a natural feature of the method applied. Indeed, this discrepancy shows that while the bifurcation was indeed detected by this method, drawing conclusions regarding its actual location should be done with caution.

It is interesting to see that except for the isolating neighborhoods that correspond to the equilibria, no other chain recurrent dynamical structures have been found. This result proves that, for the investigated ranges of parameters, the entire chain recurrent dynamics in the system restricted to the predefined set $B$ is limited to the neighborhoods of the equilibria. In particular, there are no periodic solutions or other chain recurrent dynamical structures in $B$ outside these neighborhoods.

We would like to point out the fact that although the set-oriented computations provide mathematically rigorous results, some caution must be exercised while interpreting them. For example, isolated invariant sets that form the Morse decomposition are contained in the explicitly constructed isolating neighborhoods, and the type of the set, as seen from the outside, is indicated by the Conley index. However, as was already pointed out in section 5.1, one must be cautious about claiming the actual stability of fixed points or existence of other invariant sets contained therein. Secondly, the connections found between the Morse sets at the combinatorial level (as chain of grid elements) provide an optimistic upper bound on the existing connections. In fact, it is the lack of a specific connection that can be proved using this method. In order to confirm the existence of a connecting orbit between two given Morse sets, additional work is necessary. One approach is to apply the Conley index to larger sets (e.g., two Morse sets and an outer bound for their connecting orbits) and use topological arguments. Another approach is to use numerics more directly. For example, the rigorous numerical procedures developed by the CAPD group [7] are very appropriate for this purpose (see, e.g., [41, 42]). Other authors have also developed relevant methods (e.g., [29]).

An intriguing question arises as to whether these numerical computations provide complete information about the dynamics. Indeed, some chain recurrent sets may not have been separated from each other, because the resolution was too coarse to see this separation. However, the resolutions that we chose in the set of parameters and in the phase space seem to be very fine in comparison to how these quantities can be controlled or determined for a real system, which bears some amount of uncertainty and noise. Therefore, we speculate that any additional details, which might possibly be revealed at finer resolutions, would in fact be of negligible importance for the actual applications. In particular, the shift in the parameter $\beta$ in detecting the bifurcation at $\mathcal{R}_{0}=1$ (see Figure 7 ) is natural, and one may argue that the numerical result is "more correct" than the analytic one: If different equilibria are very close together, then from the practical point of view they should be treated as a single state of the system. The reader is referred to [22] for a more in-depth discussion of this issue and for a suggestion of a theory that deals with perception of dynamics at finite resolution.

6. Conclusions. In this paper, a simple vaccination model was proposed for the spread of an infectious disease in a population of individuals who travel between two regions. We considered the three-dimensional system (1.1) as an epidemic building block in each region, and then we built our model by adding linear terms to the equations to reflect the inflow and outflow of individuals due to travel.

We calculated the basic reproduction number $\mathcal{R}_{0}$ for the two-patch model, where we 
allowed corresponding parameters in the two regions to differ. After deriving stability results for the unique DFE in terms of $\mathcal{R}_{0}$, we moved to the case of symmetric regions, to focus our attention on the impact of individuals' mobility on the steady states. In the case when the two regions are disconnected, we found out that there exist a unique componentwise positive equilibrium and two partially endemic steady states (i.e., the disease is present in one region and is absent in the other one) for $\mathcal{R}_{0}>1$. Moreover, a critical value $\mathcal{R}_{c}$ was defined such that, under certain conditions on the model parameters, the model for disconnected regions admits four fully endemic (componentwise positive) and four partially endemic equilibria for $\mathcal{R}_{c}<\mathcal{R}_{0}<1$. Stability of steady states was also described.

All partially endemic equilibria disappear when traveling between the regions is introduced into the system. For $\mathcal{R}_{0}>1$, we showed that the model for connected regions admits a single nontrivial steady state, which is stable and independent of the travel rate $\alpha$. On the other hand, the existence of multiple endemic equilibria is possible for $\mathcal{R}_{0}<1$; a necessary condition of the model parameters was given such that there are nontrivial steady states when $\mathcal{R}_{c}<\mathcal{R}_{0}<1$. In particular, the stable DFE can coexist with three stable and five unstable positive equilibria for some values of $\mathcal{R}_{0}$ on the interval $\left(\mathcal{R}_{c}, 1\right)$ if the condition for multiple steady states holds true. Two positive equilibria, bifurcating from a saddle-node bifurcation point at $\mathcal{R}_{0}=\mathcal{R}_{c}$, exist for all travel rates, while the other six endemic steady states were proven to be sensitive to the travel volume and disappear for larger values of $\alpha$. Numerical simulations were conducted to illustrate the rich bifurcation structure in the model. Using rigorous set-oriented computations, we were also able to compute a comprehensive overview of global dynamics for selected ranges of parameters. The overall image of the global dynamics agrees with the analytic results and with the numerical simulations restricted to the equilibria, and proves that outside small neighborhoods of the equilibria no other chain recurrent dynamics exists in the system.

We remark that in the case $\mathcal{R}_{0}>1$, two steady states out of the four equilibria of the disconnected system cease to exist with the introduction of traveling between the regions. In the system of connected regions, only one nontrivial steady state - a stable, fully endemic equilibrium - is preserved in addition to the unstable DFE, whereas all partially endemic equilibria disappear. This phenomenon was noted in numerous metapopulation models in the literature (see, for instance, Arino [2] and Arino and van den Driessche [3]). Its epidemiological implication is that the reproduction number, as a single quantity, plays the role of an epidemic threshold in all regions, in the sense that disease eradication in a patch is impossible as long as the infection is present in the other region and $\mathcal{R}_{0}>1$. However, bringing $\mathcal{R}_{0}$ below 1 might not be sufficient (although always necessary) for successful epidemic control: the presence of stable nontrivial equilibria implies that in some cases the disease can sustain itself even if $\mathcal{R}_{0}<1$. Based on rigorous computations on the global dynamics, the stable endemic equilibrium attracts every solution if $\mathcal{R}_{0}>1$. Since this steady state is independent of the travel rate, we conclude that spatial dispersal of individuals won't decrease the epidemic size when the reproduction number is larger than 1 . On the other hand, we showed that for $\mathcal{R}_{c}<\mathcal{R}_{0}<1$ a large number of steady states can exist in the model, and we illustrated how increasing travel volumes creates or destroys these equilibria. This rich structure of steady states goes hand in hand with some complicated dynamical behavior, which makes predicting final epidemic outcomes particularly difficult. Albeit the DFE is locally stable, its attracting 
region is complicated to characterize. For this reason, prevention strategies should focus on decreasing the reproduction number below $\mathcal{R}_{c}$, which ensures disease eradication.

Acknowledgment. The authors thank the Computer Science and Technology Center at the Department of Computer Science of the University of Minho in Portugal for providing their cluster computing facilities for the computations discussed in section 5 .

\section{REFERENCES}

[1] Z. Arai, W. Kalies, H. Kokubu, K. Mischaikow, H. Oka, and P. Pilarczyk, A database schema for the analysis of global dynamics of multiparameter systems, SIAM J. Appl. Dyn. Syst., 8 (2009), pp. 757-789.

[2] J. Arino, Diseases in metapopulations, in Modeling and Dynamics of Infectious Diseases, Ser. Contemp. Appl. Math. CAM 11, Higher Education Press, Beijing, 2009, pp. 64-122.

[3] J. Arino and P. van den Driessche, A multi-city epidemic model, Math. Popul. Stud., 10 (2003), pp. 175-193.

[4] F. Brauer, Backward bifurcations in simple vaccination models, J. Math. Anal. Appl., 298 (2004), pp. $418-431$.

[5] F. Brauer, Backward bifurcations in simple vaccination/treatment models, J. Biol. Dyn., 5 (2011), pp. 410-418.

[6] J. Bush, M. Gameiro, S. Harker, H. Kokubu, K. Mischaikow, I. Obayashi, and P. Pilarczyk, Combinatorial-topological framework for the analysis of global dynamics, Chaos, 22 (2012), 047508.

[7] The CAPD Group, Computer Assisted Proofs in Dynamics Library, Jagiellonian University, Krakow, Poland, http://capd.ii.uj.edu.pl/ (accessed on September 12, 2014).

[8] C. Conley, Isolated Invariant Sets and the Morse Index, AMS, Providence, RI, 1978.

[9] O. Diekmann and J. A. P. Heesterbeek, Mathematical Epidemiology of Infectious Diseases: Model Building, Analysis and Interpretation, Wiley, Chichester, UK, 2000.

[10] O. Diekmann, J. A. P. Heesterbeek, and J. A. J. Metz, On the definition and computation of the basic reproduction ratio $R 0$ in models for infectious diseases in heterogeneous populations, J. Math. Biol., 28 (1990), pp. 365-382.

[11] O. Diekmann, J. A. P. Heesterbeek, and M. G. Robert, The construction of next-generation matrices for compartmental epidemic models, J. R. Soc. Interface, 7 (2010), pp. 873-885.

[12] J. Dushoff, W. Huang, and C. Castillo-Chavez, Backwards bifurcations and catastrophe in simple models of total diseases, J. Math. Biol., 36 (1998), pp. 227-248.

[13] M. Gameiro, H. Kokubu, K. Mischaikow, T. Miyaji, and P. Pilarczyk, A study of rigorous ODE integrators for multi-scale set-oriented computations, in preparation, Universidade do São Paulo/Kyoto University/Rutgers/Meiji University/Institute of Science and Technology Austria.

[14] A. B. Gumel, Causes of backward bifurcations in some epidemiological models, J. Math. Anal. Appl., 395 (2012), pp. 355-365.

[15] K. P. Hadeler and C. Castillo-Chavez, A core group model for disease transmission, Math. Biosci., 128 (1995), pp. 41-55.

[16] K. P. HAdeler And P. VAn Den Driessche, Backward bifurcation in epidemic control, Math. Biosci., 146 (1997), pp. 15-35.

[17] S. Harker, H. Kokubu, K. Mischaikow, and P. Pilarczyk, Inducing a map on homology from a correspondence, Proc. Amer. Math. Soc., to appear.

[18] H. W. Нетнсоте, Qualitative analyses of communicable disease models, Math. Biosci., 28 (1976), pp. $335-356$.

[19] C. M. KRIBs-Zaleta, Center manifolds and normal forms in epidemic models, in Mathematical Approaches for Emerging and Re-emerging Infectious Diseases: An Introduction, C. Castillo-Chavez, S. Blower, D. Kirschner, P. van den Driessche, and A. A. Yakubu, eds., Springer-Verlag, New York, Berlin, 2001, pp. 269-286.

[20] C. M. Kribs-Zaleta and J. X. Velasco-Hernández, A simple vaccination model with multiple endemic states, Math. Biosci., 164 (2000), pp. 183-201. 
[21] E. Liz AND P. PilarczyK, Global dynamics in a stage-structured discrete-time population model with harvesting, J. Theoret. Biol., 297 (2012), pp. 148-165.

[22] S. Luzzatto and P. Pilarczyk, Finite resolution dynamics, Found. Comput. Math., 11 (2011), pp. 211-239.

[23] L. MARkus, Asymptotically autonomous differential systems, in Contributions to the Theory of Nonlinear Oscillations III, Ann. Math. Stud. 36, S. Lefschetz, ed., Princeton University Press, Princeton, NJ, 1956, pp. 17-29.

[24] G. F. Medley, N. A. Lindop, W. J. Edmunds, And D. J. Nokes, Hepatitis-B virus endemicity: Heterogeneity, catastrophic dynamics and control, Nature Med., 7 (2001), pp. 619-624.

[25] K. Mischaikow, M. Mrozek, and P. Pilarczyk, Graph approach to the computation of the homology of continuous maps, Found. Comput. Math., 5 (2005), pp. 199-229.

[26] R. E. Moore, Interval Analysis, Prentice-Hall, Englewood Cliffs, NJ, 1966.

[27] M. Mrozek, Leray functor and cohomological Conley index for discrete dynamical systems, Trans. Amer. Math. Soc., 318 (1990), pp. 149-178.

[28] M. MrozeK, The Conley index on compact ANR's is of finite type, Results in Math., 18 (1990), pp. 306-313.

[29] S. OisHI, Numerical verification method of existence of connecting orbits for continuous dynamical systems, J. UCS, 4 (1998), pp. 193-201.

[30] P. Pilarczyk, Computer assisted method for proving existence of periodic orbits, Topol. Methods Nonlinear Anal., 13 (1999), pp. 365-377.

[31] P. PilarczyK, Parallelization method for a continuous property, Found. Comput. Math., 10 (2010), pp. 93-114.

[32] P. PilarczyK, Rich Bifurcation Structure in a Two-Patch Vaccination Model. Results of Computations, software and computation results, 2014, http://www.pawelpilarczyk.com/infmodel/.

[33] P. Pilarczyk, L. García, B. A. Carreras, and I. Llerena, A dynamical model for plasma confinement transitions, J. Phys. A, 45 (2012), 125502.

[34] P. PilarczyK And K. Stolot, Excision-preserving cubical approach to the algorithmic computation of the discrete Conley index, Topol. Appl., 155 (2008), pp. 1149-1162.

[35] A. Szymczak, The Conley index for discrete semidynamical systems, Topol. Appl., 66 (1995), pp. 215240.

[36] H. R. Thieme, Asymptotically autonomous differential equations in the plane, Rocky Mountain J. Math., 24 (1994), pp. 351-380.

[37] H. R. Thieme and C. Castillo-Chavez, Asymptotically autonomous epidemic models, in Mathematical Population Dynamics: Analysis of Heterogeneity, Vol. I, Theory of Epidemics, O. Arino, D. Axelrod, M. Kimmel, and M. Langlais, eds., Wuerz Publishing, 1995, pp. 33-50.

[38] P. van den Driessche and J. Watmough, Reproduction numbers and sub-threshold endemic equilibria for compartmental models of disease transmission, Math. Biosci., 180 (2002), pp. 29-48.

[39] W. Wang And G. Mulone, Threshold of disease transmission in a patch environment, J. Math. Anal. App., 285 (2003), pp. 321-335.

[40] W. Wang And X.-Q. ZhaO, An epidemic model in a patchy environment, Math. Biosci., 190 (2004), pp. 97-112.

[41] D. Wilczak, Symmetric heteroclinic connections in the Michelson system: A computer assisted proof, SIAM J. Appl. Dyn. Syst., 4 (2005), pp. 489-514.

[42] D. WILCZAK AND P. ZGLICZYŃSKI, Heteroclinic connections between periodic orbits in planar restricted circular three-body problem-A computer assisted proof, Comm. Math. Phys., 234 (2003), pp. 37-75. 